\title{
OPTIMAL STOPPING PROBLEMS FOR ASSET MANAGEMENT
}

\author{
SAVAS DAYANIK, ${ }^{*}$ Bilkent University \\ MASAHIKO EGAMI, ${ }^{* *}$ Kyoto University
}

\begin{abstract}
An asset manager invests the savings of some investors in a portfolio of defaultable bonds. The manager pays the investors coupons at a constant rate and receives a management fee proportional to the value of the portfolio. He/she also has the right to walk out of the contract at any time with the net terminal value of the portfolio after payment of the investors' initial funds, and is not responsible for any deficit. To control the principal losses, investors may buy from the manager a limited protection which terminates the agreement as soon as the value of the portfolio drops below a predetermined threshold. We assume that the value of the portfolio is a jump diffusion process and find an optimal termination rule of the manager with and without protection. We also derive the indifference price of a limited protection. We illustrate the solution method on a numerical example. The motivation comes from the collateralized debt obligations.
\end{abstract}

Keywords: Optimal stopping; jump diffusion; asset management

2010 Mathematics Subject Classification: Primary 60G40

Secondary 60J60; 60J70; 60J75

\section{Introduction}

We study two optimal stopping problems of an institutional asset manager hired by ordinary investors who do not have access to certain asset classes. The investors entrust their initial funds in the amount of $L$ to the asset manager. As long as the contract is alive, the investors receive coupon payments from the asset manager on their initial funds at a fixed rate (higher than the risk-free interest rate). In return, the asset manager collects a dividend or management fee (at a fixed rate on the market value of the portfolio). At any time, the asset manager has the right to terminate the contract and walk away with the net terminal value of the portfolio after payment of the investors' initial funds. However, s/he is not financially responsible for any amount of shortfall. The asset manager's first problem is to find a nonanticipative stopping rule which maximizes her/his expected discounted total income.

Under the original contract, investors face the risk of losing all or some part of their initial funds. Suppose that the asset manager offers the investors a limited protection against this risk, in the form that the new contract will terminate as soon as the market value of the portfolio goes below a predetermined threshold. The asset manager's second problem is to find the fair price for the limited protection and the best time to terminate the contract under this additional clause.

Received 12 July 2010; revision received 14 November 2011.

* Postal address: Departments of Industrial Engineering and Mathematics, Bilkent University, 06800 Bilkent, Ankara, Turkey. Email address: sdayanik@bilkent.edu.tr

** Postal address: Graduate School of Economics, Kyoto University, Yoshida-Honmachi, Sakyo-ku, Kyoto 606-8501, Japan. Email address: egami@econ.kyoto-u.ac.jp 
We assume that the market value $X$ of the asset manager's portfolio follows a geometric Brownian motion subject to downward jumps which occur according to an independent Poisson process. As explained in detail in the next section, both the problems and the setting are motivated by those faced by the managers responsible for the portfolios of defaultable bonds, for example, as in collateralized debt obligations (CDOs). For a detailed description and the valuation of CDOs, we refer the reader to [16], [18], [19], and [23]. Briefly, a CDO is a derivative security on a portfolio of bonds, loans, or other credit risky claims. Cash flows from a collateral portfolio are divided into various quality/yield tranches which are then sold to investors. In our setting, for example, the times of the (downward) jumps in the portfolio value process can be thought of as the default times of individual bonds in the portfolio.

The difference between the real-world CDOs and our setting is that a CDO has a predetermined maturity while we assume an infinite time horizon. However, a typical CDO contract has a term of 10-15 years (much longer than, for example, finite-maturity Americantype stock options) and is often extendable with the investors' consent. Hence, our perpetuality assumption is a reasonable approximation of the reality. We believe that our analysis is also applicable in certain other financial and real-option settings with no fixed maturity, e.g. openend mutual funds and outsourcing the maintenance of computing, printing, or Internet facilities in a company or university.

To find the solutions of the asset manager's aforementioned problems, we first model them as optimal stopping problems for a suitable jump diffusion process under a risk-neutral probability measure. By separating the jumps from the diffusion part by means of a suitable dynamic programming operator, similarly to the approach used in [12] and [13] for the solutions of sequential statistics problems, we solve the optimal stopping problems by means of successive approximations, which not only lead to accurate and efficient numerical algorithms but also allow us to establish concretely the form of optimal stopping strategies. The idea of stripping the jumps from the diffusion part of a jump diffusion process was inspired by the seminal work of Davis [8], [9] on piecewise-deterministic Markov processes and the personal discussions of one of the authors with E. Çınlar (see also his talk on the web [4]).

Without any protection, the optimal rule of the asset manager turns out to terminate the contract if the market value of the portfolio $X$ becomes too small or too large, i.e. as soon as $X$ exits an interval $(a, b)$ for some suitable constants $0<a<b<\infty$.

In the presence of limited protection (provided to the investors by the asset manager for a fee) at some level $\ell \in(a, L]$, it is optimal for the asset manager to terminate the contract as soon as the value $X$ of the portfolio exits an interval $(\ell, m)$ for some suitable $m \in[\ell, b)$. Namely, if the protection is binding, i.e. $\ell \in(a, b)$, then the asset manager's optimal continuation region shrinks. In other words, investors can have limited protection only if they are also willing to give up in part on the upside potential of their managed portfolio. 'Total protection' (i.e. the case $\ell=L$ ) wipes out the upside-potential completely since the optimal strategy of the asset manager becomes 'stop immediately' in this extreme case (i.e. $\ell=m=L$ ). Incidentally, a contract with protection at some level is less valuable than an identical contract without protection. The difference between these two values gives the fair price of the investors' protection. The investors must pay this difference to the asset manager in order to compensate for the asset manager's lost potential revenues due to 'suboptimal' termination of the contract in the presence of the protection. In other words, the asset manager will be willing to provide the protection only if the difference between the expected total revenues with and without the protection is cleared by the investors. 
Our model also sheds some light on the default timing problem of a single firm. Note that the lower boundary $a$ of the optimal continuation region in the first problem's solution may be interpreted as the 'optimal default time' of a CDO. Instead of the value of a portfolio, if $X$ represents the market value of a firm subject to unexpected 'bad news' (downward jumps), then the asset manager's first problem and its solution translate into the default and sale timing problem of the firm and its solution. An action (default or sale) is optimal if the value $X$ of the firm leaves the optimal continuation region $(a, b)$. It is optimal to default if $X$ reaches $(0, a]$, and optimal to sell the firm if $X$ reaches $[b, \infty)$. Our solution extends the work of Duffie [15, Chapter 11], who calculated (based on the paper by Leland [22]) the optimal default time for a single firm whose asset value is modeled by a geometric Brownian motion.

Let us also mention that optimal stopping problems (especially pricing American-type options) for Lévy processes have been extensively studied; see, for example, [1], [3], [5], [21], [24], [25], and [26].

The problems are formulated in Section 2. The solutions to the first and second problems are studied in Sections 3 and 4, respectively. The solution methods of problems 1 and 2 are illustrated with a numerical example in Section 5.

\section{The problem description}

Let $(\Omega, \mathcal{F}, \mathrm{P})$ be a probability space hosting a Brownian motion $B=\left\{B_{t}, t \geq 0\right\}$ and an independent Poisson process $N=\left\{N_{t}, t \geq 0\right\}$ with the constant arrival rate $\lambda$, both adapted to some filtration $\mathbb{F}=\left\{\mathcal{F}_{t}\right\}_{t \geq 0}$ satisfying the usual conditions.

An asset manager borrows $L$ dollars from some investors and invests in some risky asset $X=\left\{X_{t}, t \geq 0\right\}$. The process $X$ has the dynamics

$$
\frac{\mathrm{d} X_{t}}{X_{t-}}=(\mu-\delta) \mathrm{d} t+\sigma \mathrm{d} B_{t}-y_{0}\left[\mathrm{~d} N_{t}-\lambda \mathrm{d} t\right], \quad t \geq 0
$$

for some constants $\mu \in \mathbb{R}, \sigma>0, \delta>0$, and $y_{0} \in(0,1)$. We denote by $\delta$ the dividend rate or the management fee received by the asset manager. Note that the absolute value of the relative jump sizes equals $y_{0}$, and the jumps are downwards. Therefore, the asset price

$$
X_{t}=X_{0} \exp \left\{\left(\mu-\delta-\frac{1}{2} \sigma^{2}+\lambda y_{0}\right) t+\sigma B_{t}\right\}\left(1-y_{0}\right)^{N_{t}}, \quad t \geq 0,
$$

is a geometric Brownian motion subject to downward jumps with constant relative jump sizes.

An interesting example of our setting is a portfolio of defaultable bonds as in the CDOs. Let $X_{t}$ be the value of a portfolio of $k$ defaultable bonds. After every default, the portfolio loses $y_{0}$ percent of its market value. The default times of each bond $i$ constitutes a Poisson process with the intensity rate $\lambda_{i}$ independent of others. Therefore, defaults occur at the rate $\lambda:=\sum_{i=1}^{k} \lambda_{i}$ at the level of the portfolio. The loss ratio upon a default is the same constant $y_{0}$ across the bonds. The defaulted bond is immediately sold at the market, and a bond with a similar default rate is bought using the sales proceeds. Under this assumption, defaults occur at the fixed rate $\lambda$ because the number of bonds in the portfolio is fixed at $k$. Egami and Esteghamat [18] showed that the dynamics in (1) are a good approximation of the dynamics of the aggregate value of individual defaultable bonds when priced in the 'intensity-based' modeling framework (see, e.g. [17]). The jump size $y_{0}$ on the portfolio level has to be calibrated.

Suppose that the asset manager pays the investors a coupon of $c$ percent on the face value of the initial borrowing $L$ on a continuously compounded basis. We assume that $c<\delta$. The asset manager has the right to terminate the contract at any time $\tau \in \mathbb{R}_{+}$and receive $\left(X_{\tau}-L\right)^{+}$. 
Dividend and coupon payments to the parties cease upon the termination of the contract. Let $0<r<c$ be the risk-free interest rate, and let $\delta$ be the collection of all $\mathbb{F}$-stopping times. The asset manager's first problem is to calculate his/her maximum expected discounted total income

$$
U(x):=\sup _{\tau \in \delta} \mathrm{E}_{x}^{\gamma}\left[\mathrm{e}^{-r \tau}\left(X_{\tau}-L\right)^{+}+\int_{0}^{\tau} \mathrm{e}^{-r t}\left(\delta X_{t}-c L\right) \mathrm{d} t\right], \quad x \in \mathbb{R}_{+},
$$

where $\mathrm{E}^{\gamma}$ is taken under the equivalent martingale measure $\mathrm{P}^{\gamma}$ for a specified market price $\gamma$ of the jump risk, and to find some $\tau^{*} \in \delta$ that attains the supremum (if it exists) under the condition

$$
0<r<c<\delta \text {. }
$$

In the case of real CDOs, the dividend payment is often subordinated to the coupon payment. However, since we allow the possibility that the asset manager's net running cash flow $\delta X_{t}-c L$ becomes negative, our formulation has a more stringent requirement on the asset manager than a simple subordination.

In the asset manager's second problem, the investors' assets have limited protection. In the presence of the limited protection at level $\ell>0$, the contract terminates at time $\widetilde{\tau}_{(\ell, \infty)}:=$ $\inf \left\{t \geq 0: X_{t} \notin(\ell, \infty)\right\}$ automatically. The asset manager wants to maximize his/her expected total discounted earnings as in (2), but now the supremum has to be taken over all $\mathbb{F}$-adapted stopping times $\tau \in \&$ which are less than or equal to $\widetilde{\tau}_{(\ell, \infty)}$ almost surely.

\section{The solution of the asset manager's first problem}

In the no-arbitrage pricing framework, the value of a contract contingent on the asset $X$ is the expectation of the total discounted payoff of the contract under some equivalent martingale measure. Since the dynamics of $X$ in (1) contain jumps, there is more than one equivalent martingale measure; see, e.g. [14] and [27, pp. 17-18, 77-78]. The restriction to $\mathscr{F}_{t}$ of every equivalent martingale measure $\mathrm{P}^{\gamma}$ in a large class admits a Radon-Nikodym derivative in the form

$$
\left.\frac{\mathrm{dP}^{\gamma}}{\mathrm{dP}}\right|_{\mathcal{F}_{t}}:=\eta_{t} \quad \text { and } \quad \frac{\mathrm{d} \eta_{t}}{\eta_{t-}}=\beta \mathrm{d} B_{t}+(\gamma-1)\left[\mathrm{d} N_{t}-\lambda \mathrm{d} t\right], \quad t \geq 0\left(\eta_{0}=1\right),
$$

which has the solution $\eta_{t}=\exp \left\{\beta B_{t}-\frac{1}{2} \beta^{2} t+N_{t} \log \gamma-(\gamma-1) \lambda t\right\}, t \geq 0$, for some constants $\beta \in \mathbb{R}$ and $\gamma>0$. The constants $\beta$ and $\gamma$ are known as the market price of the diffusion risk and the market price of the jump risk, respectively, and satisfy the drift conditions

$$
\gamma>0 \text { and } \mu-r+\sigma \beta-\lambda y_{0}(\gamma-1)=0 .
$$

Then the discounted value process $\left\{\mathrm{e}^{-(r-\delta) t} X_{t}: t \geq 0\right\}$ before the dividends are paid is a $\left(\mathrm{P}^{\gamma}, \mathbb{F}\right)$-martingale; see, e.g. [6], [7], and [26]. Girsanov's theorem implies that $B_{t}^{\gamma}:=B_{t}-\beta t$, $t \geq 0$, is a standard Brownian motion, and $N_{t}, t \geq 0$, is a homogeneous Poisson process with intensity $\lambda \gamma$ independent of $B^{\gamma}$ under the new measure $\mathrm{P}^{\gamma}$. Then

$$
\frac{\mathrm{d} X_{t}}{X_{t-}}=(r-\delta) \mathrm{d} t+\sigma \mathrm{d} B_{t}^{\gamma}-y_{0}\left[\mathrm{~d} N_{t}-\lambda \gamma \mathrm{d} t\right], \quad t \geq 0,
$$

where $\mu-\delta+\beta \sigma-\lambda y_{0}(\gamma-1)=r-\delta$ follows from the drift condition in (3). Itô's rule implies that

$$
X_{t}=X_{0} \exp \left\{\left(r-\delta-\frac{1}{2} \sigma^{2}+\lambda \gamma y_{0}\right) t+\sigma B_{t}^{\gamma}\right\}\left(1-y_{0}\right)^{N_{t}}, \quad t \geq 0 .
$$


The infinitesimal generator of the process $X$ under the probability measure $\mathrm{P}^{\gamma}$ coincides with the second-order differential-difference operator

$$
\left(\mathcal{A}^{\gamma} f\right)(x):=\left(r-\delta+\lambda \gamma y_{0}\right) x f^{\prime}(x)+\frac{1}{2} \sigma^{2} x^{2} f^{\prime \prime}(x)+\lambda \gamma\left[f\left(x\left(1-y_{0}\right)\right)-f(x)\right]
$$

on the collection of twice continuously differentiable functions $f(\cdot)$.

Because $\left\{\mathrm{e}^{-(r-\delta) t} X_{t}, t \geq 0\right\}$ is a martingale under $\mathrm{P}^{\gamma}$, we have

$$
\mathrm{E}_{x}^{\gamma}\left[\int_{0}^{\infty} \delta X_{t} \mathrm{e}^{-r t} \mathrm{~d} t\right]=\int_{0}^{\infty} \delta x \mathrm{e}^{-\delta t} \mathrm{~d} t=x
$$

and, for every stopping time $\tau \in \mathcal{S}$, the strong Markov property implies that

$$
\begin{aligned}
\mathrm{E}_{x}^{\gamma}\left[\int_{0}^{\tau} \delta X_{t} \mathrm{e}^{-r t} \mathrm{~d} t\right] & =\mathrm{E}_{x}^{\gamma}\left[\int_{0}^{\infty} \delta X_{t} \mathrm{e}^{-r t} \mathrm{~d} t\right]-\mathrm{E}_{x}^{\gamma}\left[\int_{\tau}^{\infty} \delta X_{t} \mathrm{e}^{-r t} \mathrm{~d} t\right] \\
& =x-\mathrm{E}_{x}^{\gamma}\left[\mathrm{e}^{-r \tau} \mathrm{E}_{X_{\tau}}^{\gamma}\left[\int_{0}^{\infty} \delta X_{s} \mathrm{e}^{-r s} \mathrm{~d} s\right]\right] \\
& =x-\mathrm{E}_{x}^{\gamma}\left[\mathrm{e}^{-r \tau} X_{\tau}\right], \quad x \in \mathbb{R}_{+} .
\end{aligned}
$$

Because $\mathrm{E}_{x}^{\gamma}\left[\int_{0}^{\tau} c L \mathrm{e}^{-r t} \mathrm{~d} t\right]=c L / r-\mathrm{E}_{x}^{\gamma}\left[c L \mathrm{e}^{-r \tau} / r\right]$ for every $\tau \in \&$ and $x \in \mathbb{R}_{+}$, (2) can be rewritten as

$$
U(x)=V(x)+x-\frac{c L}{r}, \quad x \in \mathbb{R}_{+},
$$

where

$$
V(x):=\sup _{\tau \in S} \mathrm{E}_{x}^{\gamma}\left[\mathrm{e}^{-r \tau}\left(\left(X_{\tau}-L\right)^{+}-X_{\tau}+\frac{c L}{r}\right)\right]
$$

is a discounted optimal stopping problem with terminal reward function

$$
h(x):=(x-L)^{+}-x+\frac{c L}{r}, \quad x \in \mathbb{R}_{+} .
$$

We fix the market price $\gamma$ of the jump risk, and the market price $\beta$ is determined by the drift condition in (3). In the remainder, we will describe the solution of the optimal stopping problem (5).

Let $T_{1}, T_{2}, \ldots$ be the arrival times of the process $N$. Observe that $X_{T_{n+1}}=\left(1-y_{0}\right) X_{T_{n+1}-}$ and

$$
\frac{X_{T_{n}+t}}{X_{T_{n}}}=\exp \left\{\left(r-\delta+\lambda \gamma y_{0}-\frac{\sigma^{2}}{2}\right) t+\sigma\left(B_{T_{n}+t}^{\gamma}-B_{T_{n}}^{\gamma}\right)\right\}, \quad 0 \leq t<T_{n+1}-T_{n}, n \geq 1 .
$$

Let us define, for every $n \geq 0$, the standard Brownian motion $B_{t}^{\gamma, n}:=B_{T_{n}+t}^{\gamma}-B_{T_{n}}^{\gamma}, t \geq 0$, and Poisson process $T_{k}^{(n)}:=T_{n+k}-T_{n}, k \geq 0$, respectively under $\mathrm{P}^{\gamma}$ and the one-dimensional diffusion process

$$
Y_{t}^{y, n}:=y \exp \left\{\left(r-\delta+\lambda \gamma y_{0}-\frac{\sigma^{2}}{2}\right) t+\sigma B_{t}^{\gamma, n}\right\}, \quad t \geq 0,
$$

which has the dynamics

$$
Y_{0}^{y, n}=y \quad \text { and } \quad \mathrm{d} Y_{t}^{y, n}=Y_{t}^{y, n}\left[\left(r-\delta+\lambda \gamma y_{0}\right) \mathrm{d} t+\sigma \mathrm{d} B_{t}^{\gamma, n}\right], \quad t \geq 0,
$$


and infinitesimal generator (under $\mathrm{P}_{x}^{\gamma}$ )

$$
\left(\mathcal{A}_{0}^{\gamma} f\right)(y)=\frac{\sigma^{2} y^{2}}{2} f^{\prime \prime}(y)+\left(r-\delta+\lambda \gamma y_{0}\right) y f^{\prime}(y)
$$

acting on twice continuously differentiable functions $f: \mathbb{R}_{+} \mapsto \mathbb{R}$. Then $X$ coincides with $Y^{X_{T_{n}}, n}$ on $\left[T_{n}, T_{n+1}\right)$ and jumps to $\left(1-y_{0}\right) Y_{T_{n+1}-T_{n}}^{X_{T_{n}, n}}$ at time $T_{n+1}$ for every $n \geq 0$; namely,

$$
X_{T_{n}+t}= \begin{cases}Y_{t}^{X_{T_{n}}, n}, & 0 \leq t<T_{n+1}-T_{n}, \\ \left(1-y_{0}\right) Y_{T_{n+1}-T_{n}}^{X_{T_{n}, n},} & t=T_{n+1}-T_{n} .\end{cases}
$$

For $n=0$, we will write $Y^{y, 0} \equiv Y^{y}=y \exp \left\{\left(r-\delta-\lambda \gamma y_{0}-\sigma^{2} / 2\right) t+\sigma B_{t}^{\gamma}\right\}$ and $Y^{X_{0}, 0} \equiv$ $Y^{X_{0}}$.

\subsection{A dynamic programming operator}

Let $\delta_{B}$ denote the collection of all stopping times of the diffusion process $Y^{X_{0}}$, or, equivalently, Brownian motion $B$. Let us take any arbitrary but fixed stopping time $\tau \in \delta_{B}$ and consider the following stopping strategy toward the solution of (5):

(i) on $\left\{\tau<T_{1}\right\}$ stop at time $\tau$,

(ii) on $\left\{\tau \geq T_{1}\right\}$, update $X$ at time $T_{1}$ to $X_{T_{1}}=\left(1-y_{0}\right) Y_{T_{1}}^{X_{0}}$ and continue optimally thereafter. The value of this new strategy is $\mathrm{E}_{x}^{\gamma}\left[\mathrm{e}^{-r \tau} h\left(X_{\tau}\right) \mathbf{1}_{\left\{\tau<T_{1}\right\}}+\mathrm{e}^{-r T_{1}} V\left(X_{T_{1}}\right) \mathbf{1}_{\left\{\tau \geq T_{1}\right\}}\right]$ and equals

$$
\begin{aligned}
\mathrm{E}_{x}^{\gamma}\left[\mathrm{e}^{-r \tau} h\left(Y_{\tau}^{X_{0}}\right) \mathbf{1}_{\left\{\tau<T_{1}\right\}}+\mathrm{e}^{-r T_{1}} V\left(\left(1-y_{0}\right) Y_{T_{1}}^{X_{0}}\right) \mathbf{1}_{\left\{\tau \geq T_{1}\right\}}\right] \\
\quad=\mathrm{E}_{x}^{\gamma}\left[\mathrm{e}^{-(r+\lambda \gamma) \tau} h\left(Y_{\tau}^{X_{0}}\right)+\int_{0}^{\tau} \lambda \gamma \mathrm{e}^{-(r+\lambda \gamma) t} V\left(\left(1-y_{0}\right) Y_{t}^{X_{0}}\right) \mathrm{d} t\right] .
\end{aligned}
$$

If, for every bounded function $w: \mathbb{R}_{+} \mapsto \mathbb{R}_{+}$, we introduce the operator

$$
(J w)(x):=\sup _{\tau \in S_{B}} \mathrm{E}_{x}^{\gamma}\left[\mathrm{e}^{-(r+\lambda \gamma) \tau} h\left(Y_{\tau}^{X_{0}}\right)+\int_{0}^{\tau} \lambda \gamma \mathrm{e}^{-(r+\lambda \gamma) t} w\left(\left(1-y_{0}\right) Y_{t}^{X_{0}}\right) \mathrm{d} t\right], \quad x \geq 0,
$$

then we expect the value function $V(\cdot)$ of (5) to be the unique fixed point of the operator $J$, namely, $V(\cdot)=(J V)(\cdot)$, and that $V(\cdot)$ is the pointwise limit of the successive approximations

$$
\begin{gathered}
v_{0}(x):=h(x)=(x-L)^{+}-x+\frac{c L}{r}, \quad x \geq 0 ; \\
v_{n}(x):=\left(J v_{n-1}\right)(x), \quad x \geq 0, n \geq 1 .
\end{gathered}
$$

Lemma 1. Let $w_{1}, w_{2}: \mathbb{R}_{+} \mapsto \mathbb{R}$ be bounded. If $w_{1}(\cdot) \leq w_{2}(\cdot)$ then $\left(J w_{1}\right)(\cdot) \leq\left(J w_{2}\right)(\cdot)$. If $w(\cdot)$ is a nonincreasing convex function such that $h(\cdot) \leq w(\cdot) \leq c L / r$ then $(J w)(\cdot)$ has the same properties.

The proof easily follows from the linearity of $y \mapsto Y_{t}^{y}$ for every fixed $t \geq 0$ and the definition of the operator $J$. The next proposition guarantees the existence of the unique fixed point of $J$.

Proposition 1. For every bounded $w_{1}, w_{2}: \mathbb{R}_{+} \mapsto \mathbb{R}$, we have $\left\|J w_{1}-J w_{2}\right\| \leq \lambda \gamma \| w_{1}-$ $w_{2} \| /(r+\lambda \gamma)$, where $\|w\|=\sup _{x \in \mathbb{R}_{+}}|w(x)|$; namely, $J$ acts as a contraction mapping on the bounded functions. 
Proof. Because $w_{1}(\cdot)$ and $w_{2}(\cdot)$ are bounded, $\left(J w_{1}\right)(\cdot)$ and $\left(J w_{2}\right)(\cdot)$ are finite, and, for every $\varepsilon$ and $x>0$, there are $\varepsilon$-optimal stopping times $\tau_{1}(\varepsilon, x)$ and $\tau_{2}(\varepsilon, x)$, which may depend on $\varepsilon$ and $x$, such that

$$
\begin{aligned}
\left(J w_{i}\right)(x)-\varepsilon \leq \mathrm{E}_{x}^{\gamma}[ & \mathrm{e}^{-(r+\lambda \gamma) \tau_{i}(\varepsilon, x)} h\left(Y_{\tau_{i}(\varepsilon, x)}^{X_{0}}\right) \\
& \left.+\int_{0}^{\tau_{i}(\varepsilon, x)} \lambda \gamma \mathrm{e}^{-(r+\lambda \gamma) t} w_{i}\left(\left(1-y_{0}\right) Y_{t}^{X_{0}}\right) \mathrm{d} t\right], \quad i=1,2 .
\end{aligned}
$$

Therefore,

$$
\left(J w_{1}\right)(x)-\left(J w_{2}\right)(x) \leq \varepsilon+\left\|w_{1}-w_{2}\right\| \int_{0}^{\infty} \lambda \gamma \mathrm{e}^{-(r+\lambda \gamma) t} \mathrm{~d} t=\varepsilon+\left\|w_{1}-w_{2}\right\| \frac{\lambda \gamma}{r+\lambda \gamma} .
$$

Interchanging the roles of $w_{1}(\cdot)$ and $w_{2}(\cdot)$ gives

$$
\left|\left(J w_{1}\right)(x)-\left(J w_{2}\right)(x)\right| \leq \varepsilon+\left\|w_{1}-w_{2}\right\| \frac{\lambda \gamma}{r+\lambda \gamma}
$$

for every $x>0$ and $\varepsilon>0$. Taking the supremum of both sides over $x \geq 0$ completes the proof.

Lemma 2. The sequence $\left(v_{n}\right)_{n \geq 0}$ of successive approximations is nondecreasing. Therefore, the pointwise limit $v_{\infty}(x):=\lim _{n \rightarrow \infty} v_{n}(x), x \geq 0$, exists. Every $v_{n}(\cdot), n \geq 0$, and $v_{\infty}(\cdot)$ are nonincreasing, convex, and bounded between $h(\cdot)$ and $c L / r$.

Lemma 2 follows from repeated applications of Lemma 1. Proposition 2 below shows that the unique fixed point of $J$ is the uniform limit of successive approximations.

Proposition 2. The limit $v_{\infty}(\cdot)=\lim _{n \rightarrow \infty} v_{n}(\cdot)=\sup _{n \geq 0} v_{n}(\cdot)$ is the unique bounded fixed point of operator J. Moreover,

$$
0 \leq v_{\infty}(x)-v_{n}(x) \leq \frac{c L}{r}\left(\frac{\lambda \gamma}{r+\lambda \gamma}\right)^{n}
$$

for every $x \geq 0$.

Proof. Since $v_{n}(\cdot) \nearrow v_{\infty}(\cdot)$ as $n \rightarrow \infty$, every $v_{n}(\cdot)$ is bounded from below by $((c-r) / r) L$, and $\mathrm{E}^{\gamma}\left[\int_{0}^{\tau} \mathrm{e}^{-(r+\lambda \gamma) t}((c-r) / r) L \mathrm{~d} t\right]<\infty$ for every $\tau \in \varsigma_{B}$, the monotone convergence theorem implies that

$$
\begin{aligned}
v_{\infty}(x) & =\sup _{n \geq 0} v_{n}(x) \\
& =\sup _{\tau \in S_{B}} \lim _{n \rightarrow \infty} \mathrm{E}_{x}^{\gamma}\left[\mathrm{e}^{-(r+\lambda \gamma) \tau} h\left(Y_{\tau}^{X_{0}}\right)+\int_{0}^{\tau} \lambda \gamma \mathrm{e}^{-(r+\lambda \gamma) t} v_{n}\left(\left(1-y_{0}\right) Y_{t}^{X_{0}}\right) \mathrm{d} t\right] \\
& =\sup _{\tau \in \mathcal{S}_{B}} \mathrm{E}_{x}^{\gamma}\left[\mathrm{e}^{-(r+\lambda \gamma) \tau} h\left(Y_{\tau}^{X_{0}}\right)+\int_{0}^{\tau} \lambda \gamma \mathrm{e}^{-(r+\lambda \gamma) t} v_{\infty}\left(\left(1-y_{0}\right) Y_{t}^{X_{0}}\right) \mathrm{d} t\right] \\
& =\left(J v_{\infty}\right)(x) .
\end{aligned}
$$

Thus, $v_{\infty}(\cdot)$ is the bounded fixed point of the contraction mapping $J$. Lemma 2 implies that $0 \leq v_{\infty}(\cdot)-v_{n}(\cdot)$, and

$$
\left\|v_{\infty}-v_{n}\right\|=\left\|J v_{\infty}-J v_{n-1}\right\| \leq \frac{\lambda \gamma}{r+\lambda \gamma}\left\|v_{\infty}-v_{n-1}\right\| \leq \cdots \leq\left(\frac{\lambda \gamma}{r+\lambda \gamma}\right)^{n} \frac{c L}{r}
$$

for every $n \geq 1$. 


\subsection{The solution of the optimal stopping problem in (9)}

We now solve the optimal stopping problem $J w$ in (9) for every fixed $w: \mathbb{R}_{+} \mapsto \mathbb{R}$ which satisfies the following assumption.

Assumption 1. Let $w: \mathbb{R}_{+} \mapsto \mathbb{R}$ be nonincreasing, convex, and bounded between $h(\cdot)$ and $c L / r$, and let $w(+\infty)=((c-r) / r) L$ and $w(0+)=(c / r) L$.

We will calculate the value function $(J w)(\cdot)$ and explicitly identify an optimal stopping rule. Because $w(\cdot)$ is bounded, we have

$$
\mathrm{E}_{x}^{\gamma}\left[\int_{0}^{\infty} \mathrm{e}^{-(r+\lambda \gamma) t}\left|w\left(\left(1-y_{0}\right) Y_{t}^{X_{0}}\right)\right| \mathrm{d} t\right] \leq\|w\| \int_{0}^{\infty} \mathrm{e}^{-(r+\lambda \gamma) t} \mathrm{~d} t=\frac{\|w\|}{r+\lambda \gamma}<\infty
$$

for $x \geq 0$, and, for every stopping time $\tau \in \S_{B}$, the strong Markov property of $Y^{X_{0}}$ at time $\tau$ implies that

$$
\begin{aligned}
(H w)(x) & :=\mathrm{E}_{x}^{\gamma}\left[\int_{0}^{\infty} \mathrm{e}^{-(r+\lambda \gamma) t} w\left(\left(1-y_{0}\right) Y_{t}^{X_{0}}\right) \mathrm{d} t\right] \\
& =\mathrm{E}_{x}^{\gamma}\left[\int_{0}^{\tau} \mathrm{e}^{-(r+\lambda \gamma) t} w\left(\left(1-y_{0}\right) Y_{t}^{X_{0}}\right) \mathrm{d} t\right]+\mathrm{E}_{x}^{\gamma}\left[\mathrm{e}^{-(r+\lambda \gamma) \tau}(H w)\left(Y_{\tau}^{X_{0}}\right)\right] .
\end{aligned}
$$

Therefore,

$$
\mathrm{E}_{x}^{\gamma}\left[\int_{0}^{\tau} \mathrm{e}^{-(r+\lambda \gamma) t} w\left(\left(1-y_{0}\right) Y_{t}^{X_{0}}\right) \mathrm{d} t\right]=(H w)(x)-\mathrm{E}_{x}^{\gamma}\left[\mathrm{e}^{-(r+\lambda \gamma) \tau}(H w)\left(Y_{\tau}^{X_{0}}\right)\right],
$$

and we can write the expected payoff $\mathrm{E}_{x}^{\gamma}\left[\mathrm{e}^{-(r+\lambda \gamma) \tau} h\left(Y_{\tau}^{X_{0}}\right)+\int_{0}^{\tau} \lambda \gamma \mathrm{e}^{-(r+\lambda \gamma) t} w\left(\left(1-y_{0}\right) \times\right.\right.$ $\left.\left.Y_{t}^{X_{0}}\right) \mathrm{d} t\right]$ in $(9)$ as $\lambda \gamma(H w)(x)+\mathrm{E}_{x}^{\gamma}\left[\mathrm{e}^{-(r+\lambda \gamma) \tau}\{h-\lambda \gamma(H w)\}\left(Y_{\tau}^{X_{0}}\right)\right]$ for every $\tau \in s_{B}$ and $x>0$. If we define

$$
(G w)(x):=\sup _{\tau \in \Im_{B}} \mathrm{E}_{x}^{\gamma}\left[\mathrm{e}^{-(r+\lambda \gamma) \tau}\{h-\lambda \gamma(H w)\}\left(Y_{\tau}^{X_{0}}\right)\right], \quad x>0,
$$

then the value function in (9) can be calculated by

$$
(J w)(x)=\lambda \gamma(H w)(x)+(G w)(x), \quad x>0 .
$$

We take $\mathbb{R}_{+}=[0, \infty)$ everywhere. The state 0 is a natural boundary point for the geometric Brownian motion $Y$ : it starts from 0 , then it stays there forever and cannot get into the interior of the state space with probability 1 . If it starts in the interior of its state space (namely, $\left.Y_{0} \in(0, \infty)\right)$ then it can never reach 0 . For all practical purposes, we can neglect state 0 and the values at 0 of any functions related to $Y$. For completeness, we can, for example, define $G(0)=G(0+),(H w)(0)=(H w)(0+)$, and $(J w)(0)=(J w)(0+)$.

Let us first calculate $(H w)(\cdot)$. Let $\psi(\cdot)$ and $\varphi(\cdot)$ respectively be the increasing and decreasing solutions of the second-order ordinary differential equation $\left(\mathcal{A}_{0} f\right)(y)-(r+\lambda \gamma) f(y)=0$, $y>0$, with respective boundary conditions $\psi(0+)=0$ and $\varphi(+\infty)=0$, where $\mathcal{A}_{0}$ is the infinitesimal generator in (8) of the diffusion process $Y^{X_{0}} \equiv Y^{X_{0}, 0}$. We can easily check that

$$
\psi(y)=y^{\alpha_{1}} \text { and } \varphi(y)=y^{\alpha_{0}} \text { for every } y>0,
$$

with the Wronskian

$$
W(y)=\psi^{\prime}(y) \varphi(y)-\psi(y) \varphi^{\prime}(y)=\left(\alpha_{0}+\alpha_{1}\right) y^{\alpha_{0}+\alpha_{1}-1}, \quad y>0,
$$


where $\alpha_{0}<\alpha_{1}$ are the roots of the characteristic function $g(\alpha)=\sigma^{2} \alpha(\alpha-1) / 2+(r-\delta+$ $\left.\lambda \gamma y_{0}\right) \alpha-(r+\lambda \gamma)$ of the above ordinary differential equation. Because both $g(0)<0$ and $g(1)<0$, we have

$$
\alpha_{0}<0<1<\alpha_{1} .
$$

We respectively define the hitting and exit times of the diffusion process $Y^{X_{0}}$ by

$$
\begin{gathered}
\tau_{a}:=\inf \left\{t \geq 0: Y_{t}^{X_{0}}=a\right\}, \quad a>0, \\
\tau_{a b}:=\inf \left\{t \geq 0: Y_{t}^{X_{0}} \notin(a, b)\right\}, \quad 0<a<b<\infty .
\end{gathered}
$$

We also define the operator

$$
\left(H_{a b} w\right)(x):=\mathrm{E}_{x}^{\gamma}\left[\int_{0}^{\tau_{a b}} \mathrm{e}^{-(r+\lambda \gamma) t} w\left(\left(1-y_{0}\right) Y_{t}^{X_{0}}\right) \mathrm{d} t+\mathbf{1}_{\left\{\tau_{a b}<\infty\right\}} \mathrm{e}^{-(r+\lambda \gamma) \tau_{a b}} h\left(Y_{\tau_{a b}}^{X_{0}}\right)\right],
$$

and the functions

$$
\psi_{a}(y):=\psi(y)-\frac{\psi(a)}{\varphi(a)} \varphi(y) \quad \text { and } \quad \varphi_{b}(y):=\varphi(y)-\frac{\varphi(b)}{\psi(b)} \psi(y) \quad \text { for every } y>0,
$$

which are respectively the increasing and decreasing solutions of $\left(\mathcal{A}_{0} f\right)(y)-(r+\lambda \gamma) f(y)=$ $0, a<y<b$, with respective boundary conditions $f(a)=0$ and $f(b)=0$. In terms of $W(\cdot)$ in (12), the Wronskian of $\psi_{a}(\cdot)$ and $\varphi_{a}(\cdot)$ becomes

$$
W_{a b}(y)=\psi_{a}^{\prime}(y) \varphi_{b}(y)-\psi_{a}(y) \varphi_{b}^{\prime}(y)=\left[1-\frac{\psi(a)}{\varphi(a)} \frac{\varphi(b)}{\varphi(b)}\right] W(y), \quad y>0 .
$$

Taylor and Karlin [20, Chapter 15] and Borodin and Salminen [2, pp. 3-5] proved Lemma 3 below.

Lemma 3. For every $x>0$, we have

$$
\begin{aligned}
(H w)(x) & :=\mathrm{E}_{x}^{\gamma}\left[\int_{0}^{\infty} \mathrm{e}^{-(r+\lambda \gamma) t} w\left(\left(1-y_{0}\right) Y_{t}^{X_{0}}\right) \mathrm{d} t\right] \\
& =\lim _{a \downarrow 0, b \uparrow \infty}\left(H_{a b} w\right)(x) \\
& =\varphi(x) \int_{0}^{x} \frac{2 \psi(\xi) w\left(\left(1-y_{0}\right) \xi\right)}{p^{2}(\xi) W(\xi)} \mathrm{d} \xi+\psi(x) \int_{x}^{\infty} \frac{2 \varphi(\xi) w\left(\left(1-y_{0}\right) \xi\right)}{p^{2}(\xi) W(\xi)} \mathrm{d} \xi,
\end{aligned}
$$

which is twice continuously differentiable on $\mathbb{R}_{+}$and satisfies the ordinary differential equation $\left(\mathcal{A}_{0} f\right)(x)-(r+\lambda \gamma) f(x)+w\left(\left(1-y_{0}\right) x\right)=0$.

Using the potential theoretic direct methods of Dayanik and Karatzas [11] and Dayanik [10], we now solve the optimal stopping problem $(G w)(\cdot)(10)$ with payoff function

$$
\begin{aligned}
& (h-\lambda \gamma(H w))(x) \\
& \begin{aligned}
=(x-L)^{+}-x+\frac{c L}{r}-\lambda \gamma\left[\varphi(x) \int_{0}^{x} \frac{2 \psi(\xi) w\left(\left(1-y_{0}\right) \xi\right)}{p^{2}(\xi) W(\xi)} \mathrm{d} \xi\right. \\
\left.+\psi(x) \int_{x}^{\infty} \frac{2 \varphi(\xi) w\left(\left(1-y_{0}\right) \xi\right)}{p^{2}(\xi) W(\xi)} \mathrm{d} \xi\right] \\
=(x-L)^{+}-x+\frac{c L}{r}-\frac{2 \lambda \gamma}{\sigma^{2}\left(\alpha_{1}-\alpha_{0}\right)}\left[x^{\alpha_{0}} \int_{0}^{x} \xi^{-1-\alpha_{0}} w\left(\left(1-y_{0}\right) \xi\right) \mathrm{d} \xi\right. \\
\\
\left.\quad+x^{\alpha_{1}} \int_{x}^{\infty} \xi^{-1-\alpha_{1}} w\left(\left(1-y_{0}\right) \xi\right) \mathrm{d} \xi\right]
\end{aligned}
\end{aligned}
$$


where $\psi(x)=x^{\alpha_{1}}, \varphi(x)=x^{\alpha_{0}}, p^{2}(\xi)=\sigma^{2} \xi^{2}$, and $W(\xi)=\psi^{\prime}(\xi) \varphi(\xi)-\psi(\xi) \varphi^{\prime}(\xi)=$ $\left(\alpha_{1}-\alpha_{0}\right) \xi^{\alpha_{0}+\alpha_{1}-1}$.

We observe that

$$
\begin{aligned}
0 \leq(H w)(x) & =\mathrm{E}_{x}^{\gamma}\left[\int_{0}^{\infty} \mathrm{e}^{-(r+\lambda \gamma) t} w\left(\left(1-y_{0}\right) Y_{t}^{X_{0}}\right) \mathrm{d} t\right] \\
& \leq \frac{c L}{r} \int_{0}^{\infty} \mathrm{e}^{-(r+\lambda \gamma) t} \mathrm{~d} t \\
& =\frac{c L}{r(r+\lambda \gamma)} \\
& <\infty
\end{aligned}
$$

Hence, $(h-\lambda \gamma(H w))(\cdot)$ is bounded, and because $\psi(+\infty)=\varphi(0+)=+\infty$, we have

$$
\limsup _{x \downarrow 0} \frac{(h-\lambda \gamma(H w))^{+}(x)}{\varphi(x)}=0 \quad \text { and } \quad \limsup _{x \uparrow \infty} \frac{(h-\lambda \gamma(H w))^{+}(x)}{\psi(x)}=0 .
$$

By Propositions 5.10 and 5.13 of [11], the value function $(G w)(\cdot)$ is finite; the set

$$
\Gamma[w]:=\{x>0 ;(G w)(x)=(h-\lambda \gamma(H w))(x)\}=\{x>0 ;(J w)(x)=h(x)\}
$$

is the optimal stopping region, and

$$
\tau[w]:=\inf \left\{t \geq 0: Y_{t}^{X_{0}} \in \Gamma[w]\right\}
$$

is an optimal stopping time for (10) — and for (9) because of (11). According to Proposition 5.12 of [11], we have

$$
\begin{gathered}
(G w)(x)=\varphi(x)(M w)(F(x)), \quad x \geq 0, \\
\text { and } \quad \Gamma[w]=F^{-1}(\{\zeta>0 ;(M w)(\zeta)=(L w)(\zeta)\}),
\end{gathered}
$$

where $F(x):=\psi(x) / \varphi(x)$ and $(M w)(\cdot)$ is the smallest nonnegative concave majorant on $\mathbb{R}_{+}$ of

$$
(L w)(\zeta):= \begin{cases}\frac{h-\lambda \gamma(H w)}{\varphi} \circ F^{-1}(\zeta), & \zeta>0 \\ 0, & \zeta=0 .\end{cases}
$$

To explicitly describe the form of the smallest nonnegative concave majorant $(M w)(\cdot)$ of $(L w)(\cdot)$, we will firstly identify a few useful properties of the function $(L w)(\cdot)$. Because $Y^{X_{0}} \equiv X_{0} Y^{1}$ by (7) and $w(\cdot)$ is bounded, the bounded convergence theorem implies that

$$
\lim _{x \uparrow \infty}(H w)(x)=\mathrm{E}_{1}^{\gamma}\left[\int_{0}^{\infty} \mathrm{e}^{-(r+\lambda \gamma) t} \lim _{x \uparrow \infty} w\left(\left(1-y_{0}\right) x Y_{t}^{1}\right) \mathrm{d} t\right]=\frac{w(+\infty)}{r+\lambda \gamma} \leq \frac{c L}{r}
$$

and

$$
\lim _{x \uparrow \infty}(h-\lambda \gamma(H w))(x)=\lim _{x \uparrow \infty}\left((x-L)^{+}-x+\frac{c L}{r}-\lambda \gamma(H w)(x)\right) \geq \frac{c-r}{r+\lambda \gamma} L>0 .
$$

Therefore,

$$
(L w)(+\infty)=\lim _{x \uparrow \infty} \frac{(h-\lambda \gamma(H w))(x)}{\varphi(x)}=+\infty .
$$


Note also that

$$
(L w)^{\prime}(\zeta)=\frac{\mathrm{d}}{\mathrm{d} \zeta}\left(\frac{h-\lambda \gamma(H w)}{\varphi} \circ F^{-1}(\zeta)\right)=\left[\frac{1}{F^{\prime}}\left(\frac{h-\lambda \gamma(H w)}{\varphi}\right)^{\prime}\right] \circ F^{-1}(\zeta) .
$$

Because $F(\cdot)$ is strictly increasing, we have $F^{\prime}>0$. Because $w(\cdot)$ is nonincreasing, the mapping $x \mapsto \mathrm{E}_{x}^{\gamma}\left[\int_{0}^{\infty} \mathrm{e}^{-(r+\lambda \gamma) t} w\left(\left(1-y_{0}\right) Y_{t}^{X_{0}}\right) \mathrm{d} t\right]=\mathrm{E}_{x}^{\gamma}\left[\int_{0}^{\infty} \mathrm{e}^{-(r+\lambda \gamma) t} w\left(\left(1-y_{0}\right) X_{0} Y_{t}^{1}\right) \mathrm{d} t\right]$ is decreasing. Then, for $x>L$, because $h(\cdot) \equiv c L / r$ is constant, the mapping $x \mapsto((h-$ $\lambda \gamma(H w)) / \varphi)(x)$ is increasing.

For every $0<x<L$, we can explicitly calculate

$$
\begin{aligned}
{\left[\frac{1}{F^{\prime}}\left(\frac{h-\lambda \gamma(H w)}{\varphi}\right)^{\prime}\right](x)=\frac{x^{-\alpha_{1}}}{\alpha_{1}-\alpha_{0}} } & {\left[\left(-\alpha_{0}\right) \frac{c L}{r}-\left(1-\alpha_{0}\right) x\right.} \\
& \left.-\frac{\lambda \gamma\left(-\alpha_{0}\right) \alpha_{1}}{r+\lambda \gamma} x^{\alpha_{1}} \int_{x}^{\infty} \xi^{-1-\alpha_{1}} w\left(\left(1-y_{0}\right) \xi\right) \mathrm{d} \xi\right],
\end{aligned}
$$

and because $\lim _{x \downarrow 0} x^{\alpha_{1}} \int_{x}^{\infty} \xi^{-1-\alpha_{1}} w\left(\left(1-y_{0}\right) \xi\right) \mathrm{d} \xi=w(0+) / \alpha_{1}$ and $\alpha_{1}>1$, we have

$$
\lim _{x \downarrow 0}\left[\frac{1}{F^{\prime}}\left(\frac{h-\lambda \gamma(H w)}{\varphi}\right)^{\prime}\right](x)=+\infty .
$$

Let us also study the sign of the second derivative $(L w)^{\prime \prime}(\cdot)$. For every $x \neq L$, Dayanik and Karatzas [11, p. 192] showed that

$$
(L w)^{\prime \prime}(F(x))=\frac{2 \varphi(x)}{p^{2}(x) W(x) F^{\prime}(x)}\left(\mathcal{A}_{0}-(r+\lambda \gamma)\right)(h-\lambda \gamma(H w))(x),
$$

and $\varphi(\cdot), p^{2}(\cdot), W(\cdot)$, and $F^{\prime}(\cdot)$ are positive. Therefore,

$$
\operatorname{sgn}\left[(L w)^{\prime \prime}(F(x))\right]=\operatorname{sgn}\left[\left(\mathcal{A}_{0}-(r+\lambda \gamma)\right)(h-\lambda \gamma(H w))(x)\right] .
$$

Recall from Lemma 3 that $\left(\mathcal{A}_{0}-(r+\lambda \gamma)\right)(H w)(x)=-w\left(\left(1-y_{0}\right) x\right)$ and because $h(x)=$ $(-x+c L / r) \mathbf{1}_{\{x \leq L\}}+((c-r) L / r) \mathbf{1}_{\{x>L\}}$, we have

$$
\begin{aligned}
\left(\mathcal{A}_{0}-\right. & (r+\lambda \gamma))(h-\lambda \gamma(H w))(x) \\
= & {\left[\lambda \gamma\left(1-y_{0}\right) x-(r+\lambda \gamma) \frac{c L}{r}+\lambda \gamma w\left(\left(1-y_{0}\right) x\right)\right] \mathbf{1}_{\{x \leq L\}} } \\
& +\left[\lambda \gamma w\left(\left(1-y_{0}\right) x\right)-(r+\lambda \gamma) \frac{(c-r) L}{r}\right] \mathbf{1}_{\{x>L\}} .
\end{aligned}
$$

Note that $\lim _{x \downarrow 0}\left(\mathcal{A}_{0}-(r+\lambda \gamma)\right)(h-\lambda \gamma(H w))(x)=-c L<0$ and $\lim _{x \uparrow \infty}\left(\mathcal{A}_{0}-(r+\lambda \gamma)\right)(h-$ $\lambda \gamma(H w))(x)=-(c-r) L<0$. Note also that $x \mapsto\left(\mathcal{A}_{0}-(r+\lambda \gamma)\right)(h-\lambda \gamma(H w))(x)$ is convex and continuous on $x \in(0, L)$ and $x \in(L, \infty)$. Therefore, $\left(\mathcal{A}_{0}-(r+\lambda \gamma)\right)(h-\lambda \gamma(H w))(x)$ is strictly negative in some open neighborhoods of 0 and $+\infty$, and is nonegative in the complement of the union of those open neighborhoods, whose closure contains $L$ if the union is not empty. Therefore, (16) implies that $(L w)(\zeta)$ is strictly concave in some neighborhoods of $\zeta=0$ and $\zeta=\infty$, and in the complement of their unions, whose closure contains $F(L)$ if the union is not empty, this function is convex. Earlier we also showed that $\zeta \mapsto(L w)(\zeta)$ is increasing at every $\zeta>F(L)$ and $(L w)(+\infty)=(L w)^{\prime}(0+)=+\infty$. Moreover,

$$
(L w)^{\prime}(F(L)-)-(L w)^{\prime}(F(L)+)=-\frac{L^{1-\alpha_{1}}}{\alpha_{1}-\alpha_{0}}<0
$$



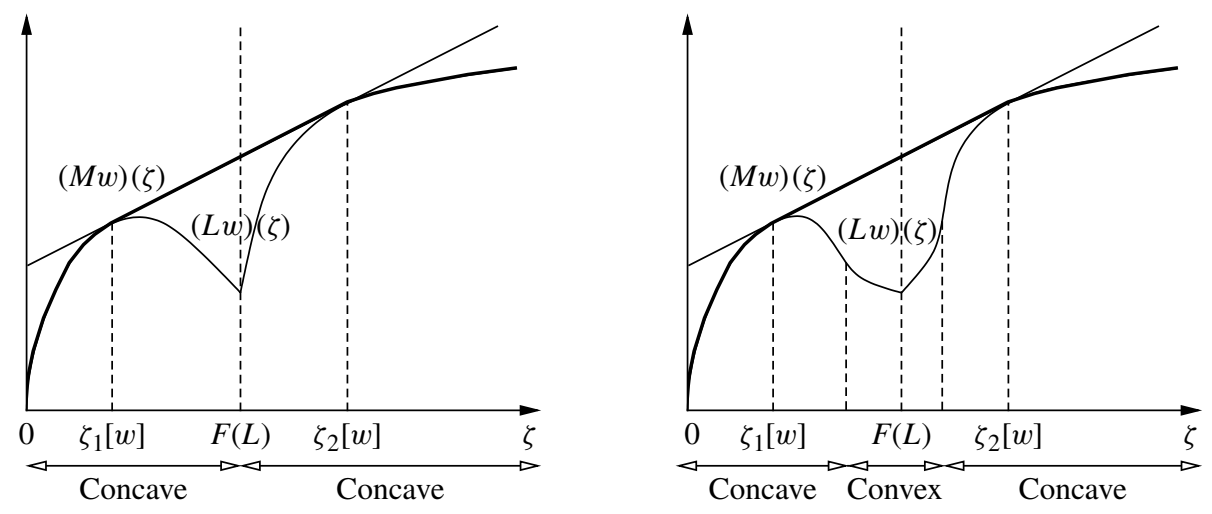

FIGURE 1: Two possible forms of $(L w)(\cdot)$ and their smallest nonnegative concave majorants $(M w)(\cdot)$.

namely, $(L w)^{\prime}(F(L)-)<(L w)^{\prime}(F(L)+)$. Two possible forms of $\zeta \mapsto(L w)(\zeta)$ and their smallest nonnegative concave majorants $\zeta \mapsto(M w)(\zeta)$ are depicted in Figure 1.

The properties of the mapping $\zeta \mapsto(L w)(\zeta)$ imply that there are unique numbers $0<$ $\zeta_{1}[w]<F(L)<\zeta_{2}[w]<\infty$ such that

$$
(L w)^{\prime}\left(\zeta_{1}[w]\right)=\frac{(L w)\left(\zeta_{2}[w]\right)-(L w)\left(\zeta_{1}[w]\right)}{\zeta_{2}[w]-\zeta_{1}[w]}=(L w)^{\prime}\left(\zeta_{2}[w]\right),
$$

and the smallest nonnegative concave majorant $(M w)(\cdot)$ of $(L w)(\cdot)$ on $\left(0, \zeta_{1}[w]\right] \cup\left[\zeta_{2}[w], \infty\right)$ coincides with $(L w)(\cdot)$, and on $\left(\zeta_{1}[w], \zeta_{2}[w]\right)$ with the straight line that majorizes $(L w)(\cdot)$ everywhere on $\mathbb{R}_{+}$and is tangent to $(L w)(\cdot)$ exactly at $\zeta=\zeta_{1}[w]$ and $\zeta_{2}[w]$; see Figure 1. More precisely,

$$
(M w)(\zeta)= \begin{cases}(L w)(\zeta), & \zeta \in\left(0, \zeta_{1}[w]\right] \cup\left[\zeta_{2}[w], \infty\right), \\ \frac{\zeta_{2}[w]-\zeta}{\zeta_{2}[w]-\zeta_{1}[w]}(L w)\left(\zeta_{1}[w]\right) & \\ \quad+\frac{\zeta-\zeta_{1}[w]}{\zeta_{2}[w]-\zeta_{1}[w]}(L w)\left(\zeta_{2}[w]\right), & \zeta \in\left(\zeta_{1}[w], \zeta_{2}[w]\right) .\end{cases}
$$

Let us define $x_{1}[w]:=F^{-1}\left(\zeta_{1}[w]\right)$ and $x_{2}[w]:=F^{-1}\left(\zeta_{2}[w]\right)$. Then, by Proposition 5.12 of [11], the value function of the optimal stopping problem in (10) equals

$$
\begin{aligned}
& (G w)(x)=\varphi(x)(M w)(F(x))
\end{aligned}
$$

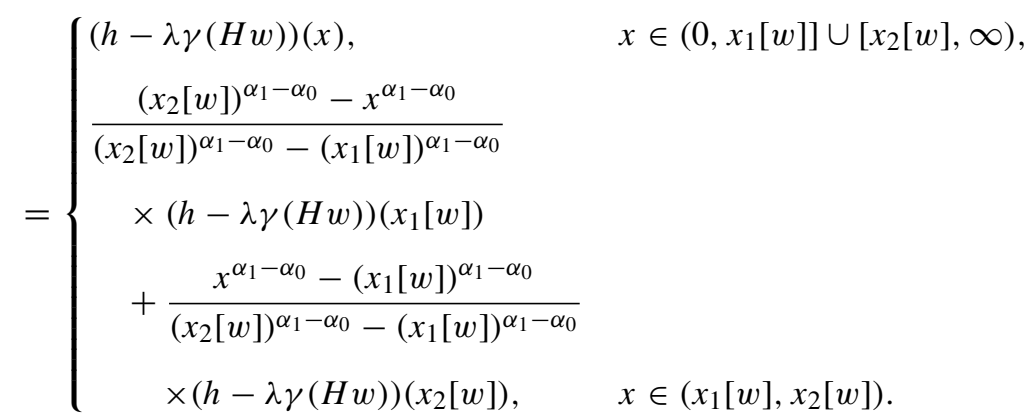


The optimal stopping region in (13) becomes

$$
\Gamma[w]=\{x>0 ;(G w)(x)=(h-\lambda \gamma)(H w)(x)\}=\left(0, x_{1}[w]\right] \cup\left[x_{2}[w], \infty\right),
$$

and the optimal stopping time in (14) becomes

$$
\tau[w]=\inf \left\{t \geq 0: Y_{t}^{X_{0}} \in\left(0, x_{1}[w]\right] \cup\left[x_{2}[w], \infty\right)\right\}
$$

Proposition 3. The value function $x \mapsto(G w)(\cdot)$ of $(10)$ is continuously differentiable on $\mathbb{R}_{+}$ and twice continuously differentiable on $\mathbb{R}_{+} \backslash\left\{x_{1}[w], x_{2}[w]\right\}$. Moreover, $(G w)(\cdot)$ satisfies

(i) $\left(\mathcal{A}_{0}-(r+\lambda \gamma)\right)(G w)(x)=0, x \in\left(x_{1}[w], x_{2}[w]\right)$,

(ii) $(G w)(x)>h(x)-\lambda \gamma(H w)(x), x \in\left(x_{1}[w], x_{2}[w]\right)$,

(iii) $\left(\mathcal{A}_{0}-(r+\lambda \gamma)\right)(G w)(x)<0, x \in\left(0, x_{1}[w]\right) \cup\left(x_{2}[w], \infty\right)$,

(iv) $(G w)(x)=h(x)-\lambda \gamma(H w)(x), x \in\left(0, x_{1}[w]\right] \cup\left[x_{2}[w], \infty\right)$.

The differentiability of $(G w)(\cdot)$ is clear from (17). The variational inequalities can be verified directly. For Proposition 3(iii), note that, if $x \in\left(0, x_{1}[w]\right) \cup\left(x_{2}[w], \infty\right)$ then $\operatorname{sgn}\left\{\left(\mathcal{A}_{0}-(r+\right.\right.$ $\lambda \gamma))(G w)(x)\}=\operatorname{sgn}\left\{\left(\mathscr{A}_{0}-(r+\lambda \gamma)\right)(h-\lambda \gamma(H w))(x)\right\}=\operatorname{sgn}\left\{(L w)^{\prime \prime}(F(x))\right\}<0$.

Because $(H w)(\cdot)$ is twice continuously differentiable and

$$
\left(\mathcal{A}_{0}-(r+\lambda \gamma)(H w)\right)(x)=-w\left(\left(1-y_{0}\right) x\right) \text { for every } x>0
$$

by Proposition 3, Proposition 3 and (11) lead directly to the next proposition.

Proposition 4. The value function $x \mapsto(J w)(\cdot)$ of $(9)$ is continuously differentiable on $\mathbb{R}_{+}$ and twice continuously differentiable on $\mathbb{R}_{+} \backslash\left\{x_{1}[w], x_{2}[w]\right\}$. Moreover, $(J w)(\cdot)$ satisfies

(i) $\left(\mathcal{A}_{0}-(r+\lambda \gamma)\right)(J w)(x)+\lambda \gamma w\left(\left(1-y_{0}\right) x\right)=0, x \in\left(x_{1}[w], x_{2}[w]\right)$,

(ii) $(J w)(x)>h(x), x \in\left(x_{1}[w], x_{2}[w]\right)$,

(iii) $\left(\mathcal{A}_{0}-(r+\lambda \gamma)\right)(J w)(x)+\lambda \gamma w\left(\left(1-y_{0}\right) x\right)<0, x \in\left(0, x_{1}[w]\right) \cup\left(x_{2}[w], \infty\right)$,

(iv) $(J w)(x)=h(x), x \in\left(0, x_{1}[w]\right] \cup\left[x_{2}[w], \infty\right)$.

By Lemma 2 , every $v_{n}(\cdot), n \geq 0$, and $v_{\infty}(\cdot)$ are nonincreasing, convex, and bounded between $h(\cdot)$ and $c L / r$. Moreover, by using induction on $n$, we can easily show that $v_{n}(0+)=$ $c L / r$ and $v_{n}(+\infty)=(c-r) L / r$ for every $n \in\{0,1, \ldots, \infty\}$. Therefore, Proposition 4, applied to $w=v_{\infty}$, and Proposition 2 directly lead to the next theorem.

Theorem 1. The function $x \mapsto v_{\infty}(x)=\left(J v_{\infty}\right)(x)$ is continuously differentiable on $\mathbb{R}_{+}$, twice continuously differentiable on $\mathbb{R}_{+} \backslash\left\{x_{1}\left[v_{\infty}\right], x_{2}\left[v_{\infty}\right]\right\}$, and satisfies the variational inequalities

(i) $\left(\mathcal{A}_{0}-(r+\lambda \gamma)\right) v_{\infty}(x)+\lambda \gamma v_{\infty}\left(\left(1-y_{0}\right) x\right)=0, x \in\left(x_{1}\left[v_{\infty}\right], x_{2}\left[v_{\infty}\right]\right)$,

(ii) $v_{\infty}(x)>h(x), x \in\left(x_{1}\left[v_{\infty}\right], x_{2}\left[v_{\infty}\right]\right)$,

(iii) $\left(\mathcal{A}_{0}-(r+\lambda \gamma)\right) v_{\infty}(x)+\lambda \gamma v_{\infty}\left(\left(1-y_{0}\right) x\right)<0, x \in\left(0, x_{1}\left[v_{\infty}\right]\right) \cup\left(x_{2}\left[v_{\infty}\right], \infty\right)$,

(iv) $v_{\infty}(x)=h(x), x \in\left(0, x_{1}\left[v_{\infty}\right]\right] \cup\left[x_{2}\left[v_{\infty}\right], \infty\right)$, 
which can be expressed in terms of the generator $\mathcal{A}^{\gamma}$ in (4) of the jump diffusion process $X$ as

$\left(\mathrm{i}^{\prime}\right)\left(\mathcal{A}^{\gamma}-r\right) v_{\infty}(x)=0, x \in\left(x_{1}\left[v_{\infty}\right], x_{2}\left[v_{\infty}\right]\right)$,

(ii') $v_{\infty}(x)>h(x), x \in\left(x_{1}\left[v_{\infty}\right], x_{2}\left[v_{\infty}\right]\right)$,

(iii') $\left(\mathcal{A}^{\gamma}-r\right) v_{\infty}(x)<0, x \in\left(0, x_{1}\left[v_{\infty}\right]\right) \cup\left(x_{2}\left[v_{\infty}\right], \infty\right)$,

$\left(\mathrm{iv}^{\prime}\right) v_{\infty}(x)=h(x), x \in\left(0, x_{1}\left[v_{\infty}\right]\right] \cup\left[x_{2}\left[v_{\infty}\right], \infty\right)$.

The next theorem identifies the value function and an optimal stopping time for the optimal stopping problem in (5). For every $w: \mathbb{R}_{+} \mapsto \mathbb{R}$ satisfying Assumption 1 , let us denote by $\tilde{\tau}[w]$ the stopping time of the jump diffusion process $X$ defined by $\tilde{\tau}[w]:=\inf \left\{t \geq 0: X_{t} \in\right.$ $\left.\left(0, x_{1}[w]\right] \cup\left[x_{2}[w], \infty\right)\right\}$.

Theorem 2. For every $x \in \mathbb{R}_{+}$, we have

$$
V(x)=v_{\infty}(x)=\mathrm{E}_{x}^{\gamma}\left[\mathrm{e}^{-r \widetilde{\tau}\left[v_{\infty}\right]} h\left(X_{\tilde{\tau}\left[v_{\infty}\right]}\right)\right],
$$

and $\tilde{\tau}\left[v_{\infty}\right]$ is an optimal stopping time for (5).

Proof. Let $\tilde{\tau}_{a b}=\inf \left\{t \geq 0: X_{t} \in(0, a] \cup[b, \infty)\right\}$ for every $0<a<b<\infty$. By Itô's rule, we have

$$
\begin{aligned}
\mathrm{e}^{-r\left(t \wedge \tau \wedge \widetilde{\tau}_{a b}\right)} v_{\infty}\left(X_{\left.t \wedge \tau \uparrow \wedge \widetilde{\tau}_{a b}\right)}\right. & \\
= & v_{\infty}\left(X_{0}\right)+\int_{0}^{t \wedge \tau \wedge \widetilde{\tau}_{a b}} \mathrm{e}^{-r s}\left(\mathcal{A}^{\gamma}-r\right) v_{\infty}\left(X_{s}\right) \mathrm{d} s+\int_{0}^{t \wedge \tau \wedge \widetilde{\tau}_{a b}} \mathrm{e}^{-r s} v_{\infty}\left(X_{s}\right) \sigma X_{s} \mathrm{~d} B_{s}^{\gamma} \\
& +\int_{0}^{t \wedge \tau \wedge \widetilde{\tau}_{a b}} \mathrm{e}^{-r s}\left[v_{\infty}\left(\left(1-y_{\infty}\right) X_{s-}\right)-v_{\infty}\left(X_{s-}\right)\right]\left(\mathrm{d} N_{s}-\lambda \gamma \mathrm{d} s\right)
\end{aligned}
$$

for every $t \geq 0, \tau \in \&$, and $0<a<b<\infty$. Because $v_{\infty}(\cdot)$ and $v_{\infty}^{\prime}(\cdot)$ are continuous and bounded on every compact subinterval of $(0, \infty)$, both stochastic integrals are square-integrable martingales, and taking the expectation of both sides gives

$$
\mathrm{E}_{x}^{\gamma}\left[\mathrm{e}^{-r\left(t \wedge \tau \wedge \widetilde{\tau}_{a b}\right)} v_{\infty}\left(X_{\left.t \wedge \tau \wedge \widetilde{\tau}_{a b}\right)}\right)=v_{\infty}(x)+\mathrm{E}_{x}^{\gamma}\left[\int_{0}^{t \wedge \tau \wedge \widetilde{\tau}_{a b}} \mathrm{e}^{-r s}\left(\mathcal{A}^{\gamma}-r\right) v_{\infty}\left(X_{s}\right) \mathrm{d} s\right] .\right.
$$

Because $\left(\mathcal{A}^{\gamma}-r\right) v_{\infty}(\cdot) \leq 0$ and $v_{\infty}(\cdot) \geq h(\cdot)$ by the variational inequalities of Theorem 1 , we have $\mathrm{E}^{\gamma}\left[\mathrm{e}^{-r\left(t \wedge \tau \wedge \widetilde{\tau}_{a b}\right)} v_{\infty}\left(X_{\left.t \wedge \tau \wedge \widetilde{\tau}_{a b}\right)}\right) \leq v_{\infty}(x)\right.$ for every $t \geq 0, \tau \in \delta$, and $0<a<b<\infty$. Because $\lim _{a \downarrow 0, b \uparrow \infty} \tilde{\tau}_{a b}=\infty$ almost surely and $h(\cdot)$ is continuous and bounded, we can take limits of both sides as $t \uparrow \infty, a \downarrow 0$, and $b \uparrow \infty$, and use the bounded convergence theorem to obtain $\mathrm{E}^{\gamma}\left[\mathrm{e}^{-r \tau} v_{\infty}\left(X_{\tau}\right)\right] \leq v_{\infty}(x)$ for every $\tau \in \S$. Taking the supremum over all $\tau \in \&$ gives $V(x)=\sup _{\tau \in \delta} \mathrm{E}^{\gamma}\left[\mathrm{e}^{-r \tau} v_{\infty}\left(X_{\tau}\right)\right] \leq v_{\infty}(x)$.

In order to show the reverse inequality, we replace $\tau$ and $\tilde{\tau}_{a b}$ in (18) with $\tilde{\tau}\left[v_{\infty}\right]$. Because $\left(\mathcal{A}^{\gamma}-r\right) v_{\infty}(x)=0$ for every $x \underset{\infty}{\in}\left(x_{1}\left[v_{\infty}\right], x_{2}\left[v_{\infty}\right]\right)$ by Theorem $1\left(\mathrm{i}^{\prime}\right), \mathrm{E}_{x}^{\gamma}\left[\mathrm{e}^{-r\left(t \wedge \tilde{\tau}\left[v_{\infty}\right]\right)} \times\right.$ $\left.v_{\infty}\left(X_{t \wedge \tilde{\tau}\left[v_{\infty}\right]}\right)\right]=v_{\infty}(x)+\mathrm{E}_{x}^{\gamma}\left[\int_{0}^{t \wedge \tau}\left[v_{\infty}\right] \mathrm{e}^{-r s}\left(\mathcal{A}^{\gamma}-r\right) v_{\infty}\left(X_{s}\right) \mathrm{d} s\right]=v_{\infty}(x)$ for every $t \geq 0$. Since $v_{\infty}$ is bounded and continuous, taking limits as $t \uparrow \infty$ and the bounded convergence give $v_{\infty}(x)=\mathrm{E}_{x}^{\gamma}\left[\mathrm{e}^{-r \widetilde{\tau}\left[v_{\infty}\right]} v_{\infty}\left(X_{\widetilde{\tau}\left[v_{\infty}\right]}\right)\right]=\mathrm{E}_{x}^{\gamma}\left[\mathrm{e}^{-r \widetilde{\tau}\left[v_{\infty}\right]} h\left(X_{\widetilde{\tau}\left[v_{\infty}\right]}\right)\right] \leq V(x)$ by Theorem $1\left(\mathrm{iv}^{\prime}\right)$, which completes the proof.

Proposition 5. The optimal stopping regions

$$
\Gamma\left[v_{n}\right]=\left\{x>0 ;\left(J v_{n}\right)(x) \leq h(x)\right\}=\left(0, x_{1}\left[v_{n}\right]\right] \cup\left[x_{2}\left[v_{n}\right], \infty\right), \quad n \in\{0,1, \ldots, \infty\},
$$


are decreasing, and $0<x_{1}\left[v_{\infty}\right] \leq \cdots \leq x_{1}\left[v_{1}\right] \leq x_{1}\left[v_{0}\right] \leq L \leq x_{2}\left[v_{0}\right] \leq x_{2}\left[v_{1}\right] \leq \cdots \leq$ $x_{2}\left[v_{\infty}\right]<\infty$. Moreover, $x_{1}\left[v_{\infty}\right]=\lim _{n \rightarrow \infty} x_{1}\left[v_{n}\right]$ and $x_{2}\left[v_{\infty}\right]=\lim _{n \rightarrow \infty} x_{2}\left[v_{n}\right]$.

The proof follows from the monotonicity of the operator $J$ and the fact that $v_{n}(x) \uparrow v_{\infty}(x)$ as $n \rightarrow \infty$ uniformly in $x>0$. The next proposition and its corollary identify the optimal expected reward and nearly optimal stopping strategies for the asset manager in the first problem.

Proposition 6. For every $n \geq 0$, we have

$$
v_{\infty}(x) \leq \mathrm{E}_{x}^{\gamma}\left[\mathrm{e}^{-r \widetilde{\tau}\left[v_{n}\right]} h\left(X_{\widetilde{\tau}\left[v_{n}\right]}\right)\right]+\frac{c L}{r}\left(\frac{\lambda}{r+\lambda \gamma}\right)^{n+1} .
$$

Hence, for every $\varepsilon>0$ and $n \geq 0$ such that $(c L / r)(\lambda /(r+\lambda \gamma))^{n+1} \leq \varepsilon$, the stopping time $\tilde{\tau}\left[v_{n}\right]$ is $\varepsilon$-optimal for (5).

Proof. Recall that $\tilde{\tau}\left[v_{n}\right]=\inf \left\{t \geq 0: X_{t} \in \Gamma\left[v_{n}\right]\right\}=\inf \left\{t \geq 0: X_{t} \in\left(0, x_{1}\left[v_{n}\right]\right] \cup\right.$ $\left[x_{2}\left[v_{n}\right], \infty\right)$. If we replace $\tau$ and $\tilde{\tau}_{a b}$ in (18) with $\tilde{\tau}\left[v_{n}\right]$ then, for every $t \geq 0$, we obtain $\mathrm{E}_{x}^{\gamma}\left[\mathrm{e}^{-r\left(t \wedge \tilde{\tau}\left[v_{n}\right]\right)} v_{\infty}\left(X_{t \wedge \tilde{\tau}\left[v_{n}\right]}\right)\right]=v_{\infty}(x)+\mathrm{E}_{x}^{\gamma}\left[\int_{0}^{t \wedge \tilde{\tau}\left[v_{n}\right]} \mathrm{e}^{-r s}\left(\mathcal{A}^{\gamma}-r\right) v_{\infty}\left(X_{s}\right) \mathrm{d} s\right]=v_{\infty}(x)$, because, for every $0<t<\tilde{\tau}\left[v_{n}\right]$, we have

$$
X_{t} \in\left(x_{1}\left[v_{n}\right], x_{2}\left[v_{n}\right]\right) \subseteq\left(x_{1}\left[v_{\infty}\right], x_{2}\left[v_{\infty}\right]\right),
$$

at every element $x$ for which $\left(\mathcal{A}^{\gamma}-r\right) v_{\infty}(x)$ equals 0 according to Theorem $1\left(\mathrm{i}^{\prime}\right)$. Because $v_{\infty}(\cdot)$ is continuous and bounded, taking limits as $t \uparrow \infty$ and the bounded convergence theorem give $v_{\infty}(x)=\mathrm{E}_{x}^{\gamma}\left[\mathrm{e}^{-r \widetilde{\tau}\left[v_{n}\right]} v_{\infty}\left(X_{\widetilde{\tau}\left[v_{n}\right]}\right)\right]$. Because $\left(J v_{n}\right)(\cdot)=h(\cdot)$ on $\Gamma\left[v_{n}\right] \ni X_{\widetilde{\tau}\left[v_{n}\right]}$ and $\left\{\tilde{\tau}\left[v_{n}\right]<\infty\right\}$, Proposition 2 implies that

$$
\begin{aligned}
v_{\infty}(x) & \leq \mathrm{E}_{x}^{\gamma}\left[\mathrm{e}^{-r \widetilde{\tau}\left[v_{n}\right]}\left(v_{n+1}\left(X_{\tilde{\tau}\left[v_{n}\right]}\right)+\frac{c L}{r}\left(\frac{\lambda \gamma}{r+\lambda \gamma}\right)^{n+1}\right)\right] \\
& \leq \mathrm{E}_{x}^{\gamma}\left[\mathrm{e}^{-r \widetilde{\tau}\left[v_{n}\right]}\left(\left(J v_{n}\right)\left(X_{\widetilde{\tau}\left[v_{n}\right]}\right)\right)\right]+\frac{c L}{r}\left(\frac{\lambda \gamma}{r+\lambda \gamma}\right)^{n+1} \\
& =\mathrm{E}_{x}^{\gamma}\left[\mathrm{e}^{-r \widetilde{\tau}\left[v_{n}\right]}\left(h\left(X_{\tilde{\tau}\left[v_{n}\right]}\right)\right)\right]+\frac{c L}{r}\left(\frac{\lambda \gamma}{r+\lambda \gamma}\right)^{n+1} .
\end{aligned}
$$

Corollary 1. The maximum expected reward of the asset manager is given by $U(x)=x-$ $c L / r+V(x)=x-c L / r+v_{\infty}(x)$ for every $x \geq 0$. The stopping rule $\tilde{\tau}\left[v_{\infty}\right]$ is optimal, and $\tilde{\tau}\left[v_{n}\right]$ is $\varepsilon$-optimal for every $\varepsilon>0$ and $n \geq 0$ such that $(c L / r)(\lambda \gamma /(r+\lambda \gamma))^{n+1}<\varepsilon$ :

$$
U(x)=\mathrm{E}_{x}^{\gamma}\left[\mathrm{e}^{-r \widetilde{\tau}\left[v_{\infty}\right]}\left(X_{\widetilde{\tau}\left[v_{\infty}\right]}-L\right)^{+}+\int_{0}^{\widetilde{\tau}\left[v_{\infty}\right]} \mathrm{e}^{-r t}\left(\delta X_{t}-c L\right) \mathrm{d} t\right]
$$

and

$$
U(x)-\varepsilon \leq \mathrm{E}_{x}^{\gamma}\left[\mathrm{e}^{-r \tilde{\tau}\left[v_{n}\right]}\left(X_{\tilde{\tau}\left[v_{n}\right]}-L\right)^{+}+\int_{0}^{\widetilde{\tau}\left[v_{n}\right]} \mathrm{e}^{-r t}\left(\delta X_{t}-c L\right) \mathrm{d} t\right], \quad x>0 .
$$

\section{The solution of the asset manager's second problem}

In the asset manager's second problem, the investors' assets have limited protection. In the presence of limited protection at level $\ell>0$, the contract terminates at time $\tilde{\tau}_{\ell, \infty}:=$ $\inf \left\{t \geq 0: X_{t} \notin(\ell, \infty)\right\}$ automatically. The asset manager wants to maximize her expected total discounted earnings as in (2), but now the supremum has to be taken over all stopping 
times $\tau \in \S$ which are less than or equal to $\tilde{\tau}_{\ell, \infty}$ almost surely. Namely, we would like to solve the problem

$$
U_{\ell}(x):=\sup _{\tau \in S} \mathrm{E}_{x}^{\gamma}\left[\mathrm{e}^{-r\left(\tilde{\tau}_{\ell, \infty} \wedge \tau\right)}\left(X_{\tilde{\tau}_{\ell, \infty} \wedge \tau}-L\right)^{+}+\int_{0}^{\tilde{\tau}_{\ell, \infty} \wedge \tau} \mathrm{e}^{-r t}\left(\delta X_{t}-c L\right) \mathrm{d} t\right]
$$

for every $x \in \mathbb{R}_{+}$. If $\ell<x_{1}\left[v_{\infty}\right]$ then $U_{\ell}(x)=U(x)=\mathrm{E}_{x}^{\gamma}\left[\mathrm{e}^{-r\left(\widetilde{\tau}\left[v_{\infty}\right]\right)}\left(X_{\tilde{\tau}_{\ell, \infty}\left[v_{\infty}\right]}-L\right)^{+}+\right.$ $\left.\int_{0}^{\widetilde{\tau}\left[v_{\infty}\right]} \mathrm{e}^{-r t}\left(\delta X_{t}-c L\right) \mathrm{d} t\right]$ for every $x>0$. On the one hand, because, for every $\tau \in \delta$, $\widetilde{\tau}\left[v_{\infty}\right] \wedge \tau$ also belongs to $\delta$, we have $U_{\ell}(x) \leq U(x)$. On the other hand, because $\ell \leq x_{1}\left[v_{\infty}\right]$, we have, almost surely, $\tilde{\tau}\left[v_{\infty}\right]=\tilde{\tau}_{\ell, \infty} \wedge \tilde{\tau}\left[v_{\infty}\right] \in \delta$ and $U_{\ell}(x) \geq \mathrm{E}_{x}^{\gamma}\left[\mathrm{e}^{-r \widetilde{\tau}\left[v_{\infty}\right]}\left(X_{\widetilde{\tau}\left[v_{\infty}\right]}-L\right)^{+}+\right.$ $\left.\int_{0}^{\widetilde{\tau}\left[v_{\infty}\right]} \mathrm{e}^{-r t}\left(\delta X_{t}-c L\right) \mathrm{d} t\right]=U(x)$ for every $x$. Therefore, $U_{\ell}(x)=U(x)$ for every $x>0$ if $\ell \leq x_{1}\left[v_{\infty}\right]$.

In the remainder of the paper, we will make the following assumption.

Assumption 2. The protection level $\ell$ is such that $x_{1}\left[v_{\infty}\right]<\ell \leq L$.

The strong Markov property of $X$ can be used to similarly show that

$$
U_{\ell}(x)=x-\frac{c L}{r}+V_{\ell}(x), \quad x \geq 0,
$$

where

$$
V_{\ell}(x):=\sup _{\tau \in S} \mathrm{E}_{x}^{\gamma}\left[\mathrm{e}^{-r\left(\widetilde{\tau}_{\ell, \infty} \wedge \tau\right)} h\left(X_{\widetilde{\tau}_{\ell, \infty} \wedge \tau}\right)\right], \quad x>0,
$$

is the discounted optimal stopping problem for the stopped jump diffusion process $X_{\widetilde{\tau}_{\ell, \infty} \wedge t}$, $t \geq 0$, with the same terminal payoff function $h(\cdot)$ as in (6). Let us define the stopping time $\tau_{\ell, \infty}:=\inf \left\{t \geq 0: Y_{t}^{X_{0}} \notin(\ell, \infty)\right\}$ of the diffusion process $Y^{X_{0}}$ and the operator

$$
\begin{aligned}
&\left(J_{\ell} w\right)(x):= \sup _{\tau \in S_{B}} \mathrm{E}_{x}^{\gamma}\left[\mathrm{e}^{-r \tau} h\left(X_{\tau_{\ell, \infty} \wedge \tau}\right) \mathbf{1}_{\left\{\tau_{\ell, \infty} \wedge \tau<T_{1}\right\}}+\mathrm{e}^{-r T_{1}} w\left(X_{T_{1}}\right) \mathbf{1}_{\left\{\tau_{\ell, \infty} \wedge \tau \geq T_{1}\right\}}\right] \\
&=\sup _{\tau \in S_{B}} \mathrm{E}_{x}^{\gamma}\left[\mathrm{e}^{-(r+\lambda \gamma)\left(\tau_{\ell, \infty} \wedge \tau\right)} h\left(Y_{\tau_{\ell, \infty} X_{0}}\right)\right. \\
&\left.\quad+\int_{0}^{\tau_{\ell, \infty} \wedge \tau} \lambda \gamma \mathrm{e}^{-(r+\lambda \gamma) t} w\left(\left(1-y_{0}\right) Y_{t}^{X_{0}}\right) \mathrm{d} t\right]
\end{aligned}
$$

for every $x \geq 0$. We expect that $V_{\ell}(\cdot)=\left(J V_{\ell}\right)(\cdot)$, namely, that $V_{\ell}(\cdot)$ is one of the fixed points of the operator $J_{\ell}$. We can find one of the fixed points of $J_{\ell}$ by taking the limit of successive approximations defined by

$$
v_{\ell, 0}(x):=h(x) \quad \text { and } \quad v_{\ell, n}(x):=\left(J_{\ell} v_{\ell, n-1}\right)(x), \quad n \geq 1, x>0 .
$$

Lemmas 1 and 2 and Propositions 1 and 2 hold with obvious changes. Let $w: \mathbb{R}_{+} \mapsto \mathbb{R}$ be a function as in Assumption 1. Then

$$
\left(J_{\ell} w\right)(x)=\lambda \gamma(H w)(x)+\left(G_{\ell} w\right)(x), \quad x>0,
$$

where

$$
\left(G_{\ell} w\right)(x):=\sup _{\tau \in S_{B}} \mathrm{E}_{x}^{\gamma}\left[\mathrm{e}^{-(r+\lambda \gamma) \tau_{\ell, \infty} \wedge \tau}\{h-\lambda \gamma(H w)\}\left(Y_{\tau_{\ell, \infty} \wedge \tau}^{X_{0}}\right)\right], \quad x>0 .
$$

We obviously have $\left(G_{\ell} w\right)(x)=h(x)-\lambda \gamma(H w)(x)$ for every $x \in(0, \ell]$. If the initial state 
$X_{0}$ of $Y_{\tau_{\ell, \infty} \wedge t}^{X_{0}}, t \geq 0$, is in $(\ell, \infty)$ then $\ell$ becomes an absorbing left boundary for the stopped process $Y_{\tau_{\ell, \infty} \wedge t}^{X_{0}}, t \geq 0$.

Let $\left(M_{\ell} w\right)(\cdot)$ be the smallest concave majorant on $[F(\ell), \infty)$ of $(L w)(\cdot)$ defined by $(15)$ and identically equal to $(L w)(\cdot)$ on $(0, F(\ell))$. Then, by Proposition 5.5 of [11], $\left(G_{\ell} w\right)(x)=$ $\varphi(x)\left(M_{\ell} w\right)(F(x)), x>0$, and $\Gamma_{\ell}[w]=F^{-1}\left(\left\{\zeta>0 ;\left(M_{\ell} w\right)(\zeta)=(L w)(\zeta)\right\}\right)$ are the value function and optimal stopping region for (21). The analysis of the shape of $(L w)(\cdot)$ prior to Figure 1 implies that there are unique numbers $0<\zeta_{\ell, 1}[w]<F(L)<\zeta_{\ell, 2}[w]<\infty$ such that, if $F(\ell) \leq \zeta_{1}[w]$,

$$
(L w)^{\prime}\left(\zeta_{\ell, 1}[w]\right)=\frac{(L w)\left(\zeta_{\ell, 2}[w]\right)-(L w)\left(\zeta_{\ell, 1}[w]\right)}{\zeta_{\ell, 2}[w]-\zeta_{\ell, 1}[w]}=(L w)^{\prime}\left(\zeta_{\ell, 2}[w]\right),
$$

namely, $\zeta_{\ell, 1}[w] \equiv \zeta_{1}[w]$ and $\zeta_{\ell, 2}[w] \equiv \zeta_{2}[w]$; if $F(\ell)>\zeta_{1}[w]$,

$$
\zeta_{\ell, 1}[w]=\ell \quad \text { and } \quad \frac{(L w)\left(\zeta_{\ell, 2}[w]\right)-(L w)\left(\zeta_{\ell, 1}[w]\right)}{\zeta_{\ell, 2}[w]-\zeta_{\ell, 1}[w]}=(L w)^{\prime}\left(\zeta_{\ell, 2}[w]\right) ;
$$

and

$$
\left(M_{\ell} w\right)(\zeta)= \begin{cases}(L w)(\zeta), & \zeta \in\left(0, \zeta_{\ell, 1}[w]\right] \cup\left[\zeta_{\ell, 2}[w], \infty\right), \\ \frac{\zeta_{\ell, 2}[w]-\zeta}{\zeta_{\ell, 2}[w]-\zeta_{\ell, 1}[w]}(L w)\left(\zeta_{\ell, 1}[w]\right) & \\ +\frac{\zeta-\zeta_{\ell, 1}[w]}{\zeta_{\ell, 2}[w]-\zeta_{\ell, 1}[w]}(L w)\left(\zeta_{\ell, 2}[w]\right), & \zeta \in\left(\zeta_{\ell, 1}[w], \zeta_{\ell, 2}[w]\right) .\end{cases}
$$

Let us define $x_{\ell, 1}[w]=F^{-1}\left(\zeta_{\ell, 1}[w]\right)$ and $x_{\ell, 2}[w]=F^{-1}\left(\zeta_{\ell, 2}[w]\right)$. Then the value function equals

$$
\begin{aligned}
& \left(G_{\ell} w\right)(x)=\varphi(x)\left(M_{\ell} w\right)(F(x)) \\
& =\left\{\begin{array}{cc}
(h-\lambda \gamma(H w))(x), & x \in\left(0, x_{\ell, 1}[w]\right] \cup\left[x_{\ell, 2}[w], \infty\right), \\
\frac{\left(x_{\ell, 2}[w]\right)^{\alpha_{1}-\alpha_{0}}-x^{\alpha_{1}-\alpha_{0}}}{\left(x_{\ell, 2}[w]\right)^{\alpha_{1}-\alpha_{0}}-\left(x_{\ell, 1}[w]\right)^{\alpha_{1}-\alpha_{0}}} & \\
\times(h-\lambda \gamma(H w))\left(x_{\ell, 1}[w]\right) & \\
+\frac{x^{\alpha_{1}-\alpha_{0}}-\left(x_{\ell, 1}[w]\right)^{\alpha_{1}-\alpha_{0}}}{\left(x_{\ell, 2}[w]\right)^{\alpha_{1}-\alpha_{0}}-\left(x_{\ell, 1}[w]\right)^{\alpha_{1}-\alpha_{0}}} & \\
\times(h-\lambda \gamma(H w))\left(x_{\ell, 2}[w]\right), & \text { otherwise, }
\end{array}\right.
\end{aligned}
$$

and the optimal stopping region and an optimal stopping time are given by

$$
\begin{aligned}
\Gamma_{\ell}[w] & =\left\{x>0 ;\left(G_{\ell} w\right)(x)=(h-\lambda \gamma(H w))(x)\right\} \\
& =\left(0, x_{\ell, 1}[w]\right] \cup\left[x_{\ell, 2}[w], \infty\right), \\
\tau_{\ell}[w] & :=\inf \left\{x>0: Y_{t}^{X_{0}} \in \Gamma_{\ell}[w]\right\} \\
& =\inf \left\{x>0: Y_{t}^{X_{0}} \in\left(0, x_{\ell, 1}[w]\right] \cup\left[x_{\ell, 2}[w], \infty\right)\right\},
\end{aligned}
$$

for the problem in (21). A direct verification, together with the chain of equalities

$$
\begin{aligned}
\operatorname{sgn}\left\{\left(\mathcal{A}_{0}-(r+\lambda \gamma)\right)\left(G_{\ell} w\right)(x)\right\} & =\operatorname{sgn}\left\{\left(\mathcal{A}_{0}-(r+\lambda \gamma)\right)(h-\lambda \gamma(H w))(x)\right\} \\
& =\operatorname{sgn}\left\{(L w)^{\prime \prime}(F(x))\right\} \\
& <0 \text { for every } x \in\left(\ell, x_{\ell, 1}[w]\right) \cup\left(x_{\ell, 2}[w], \infty\right)
\end{aligned}
$$


from [11, p. 192], proves Propositions 3 and 4 and Theorem 1 for the second problem with $G$, $H, J$ replaced by $G_{\ell}, H_{\ell}, J_{\ell}$ and all functions restricted to $[\ell, \infty)$. By the next theorem, the optimal stopping time for the asset manager's second problem is of the form $\tilde{\tau}_{\ell}[w]:=\inf \{t \geq$ $\left.0: X_{t} \in\left(0, x_{\ell, 1}[w]\right] \cup\left[x_{\ell, 2}[w], \infty\right)\right\}$.

Theorem 3. For every $x \in \mathbb{R}_{+}$, we have

$$
V_{\ell}(x)=v_{\ell, \infty}(x)=\mathrm{E}_{x}^{\gamma}\left[\mathrm{e}^{-r \tilde{\tau}_{\ell}\left[v_{\ell, \infty}\right]} h\left(X_{\tilde{\tau}_{\ell}\left[v_{\ell, \infty}\right]}\right)\right],
$$

and $\tilde{\tau}_{\ell}\left[v_{\ell, \infty}\right]$ is an optimal stopping time for (19).

The proof is similar to that of Theorem 2, and Propositions 5 and 6 and Corollary 1 hold with obvious changes. We expect the value of the limited protection at level $\ell$ to increase as $\ell$ increases. We also expect that the asset manager quits early as the protection limit $\ell$ increases to $L$. Such expectations are validated by means of the next lemma.

Lemma 4. Let $w: \mathbb{R}_{+} \mapsto \mathbb{R}$ be as in Assumption 1. Suppose that $0<\ell<u<L$. Then

(i) $\left(M_{\ell} w\right)(\cdot) \geq\left(M_{u} w\right)(\cdot)$ on $\mathbb{R}_{+}$,

(ii) $0<\zeta_{\ell, 1}[w]<\zeta_{u, 1}[w]<F(L)<\zeta_{u, 2}[w]<\zeta_{\ell, 2}[w]<\infty$,

(iii) $\left(J_{\ell} w\right)(\cdot) \geq\left(J_{u} w\right)(\cdot)$ on $\mathbb{R}_{+}$,

(iv) $0<x_{\ell, 1}[w]<x_{u, 1}[w]<L<x_{u, 2}[w]<x_{\ell, 2}[w]<\infty$.

Recall that $\left(M_{\ell} w\right)(\cdot)$ and $\left(M_{u} w\right)(\cdot)$ coincide on $(0, F(\ell)]$ and $(0, F(u)]$, respectively, with $(L w)(\cdot)$ and on $(F(\ell), \infty)$ and $(F(u), \infty)$, respectively, with the smallest nonnegative concave majorants of $(L w)(\cdot)$ over $(F(\ell), \infty)$ and $(F(u), \infty)$, respectively. Therefore, (i) and (ii) of Lemma 4 immediately follow; see the bottom diagram of Figure 2. Finally, Lemma 4(iii) and (iv) follow from (i) and (ii) by (20), i.e. $\left(J_{\ell} w\right)(x)=\lambda \gamma(H w)(x)+\left(G_{\ell} w\right)(x)=\lambda \gamma(H w)(x)+$ $\varphi(x)\left(M_{\ell} w\right)(F(x))$ for every $x, x_{\ell, 1}[w]=F^{-1}\left(\zeta_{\ell, 1}[w]\right), x_{\ell, 2}[w]=F^{-1}\left(\zeta_{\ell, 2}[w]\right)$, and the fact that $F(\cdot)$ is strictly increasing.

Proposition 7 shows that demanding higher portfolio insurance or limiting more severely the downward risks or losses also limits the upward potential and reduces the portfolio's total value.

Proposition 7. For every $0<\ell<u<L$,

(i) $v_{\ell, n}(x) \geq v_{u, n}(x)$ for all $0 \leq n \leq \infty$,

(ii) $U_{\ell}(x) \geq U_{u}(x)$ for every $x \in \mathbb{R}_{+}$, and

(iii) $0<x_{\ell, 1}\left[v_{\ell, n}\right] \leq x_{u, 1}\left[v_{u, n}\right]<L<x_{u, 2}\left[v_{u, n}\right] \leq x_{\ell, 2}\left[v_{\ell, n}\right]<\infty$.

Proof. Note first that $v_{\ell, 0}(x)=h(x)=v_{u, 0}(x)$ for every $x \in \mathbb{R}_{+}$. Suppose that $v_{\ell, n}(\cdot) \geq$ $v_{u, n}(\cdot)$ for some $n \geq 0$. Then, by monotonicity and Lemma 4(iii), $v_{\ell, n+1}(\cdot)=\left(J_{\ell} v_{\ell, n}\right)(\cdot) \geq$ $\left(J_{\ell} v_{u, n}\right)(\cdot) \geq\left(J_{u} v_{u, n}\right)(\cdot)=v_{u, n+1}(\cdot)$. Therefore, for every $n \geq 0, v_{\ell, n}(\cdot) \geq v_{u, n}(\cdot)$ and $v_{\ell, \infty}(\cdot)=\lim _{n \rightarrow \infty} v_{\ell, n}(\cdot) \geq \lim _{n \rightarrow \infty} v_{u, n}(\cdot)=v_{u, \infty}(\cdot)$, which proves (i). By (19), $U_{\ell}(x)=$ $x-c L / r+v_{\ell, \infty}(x) \geq x-c L / r+v_{u, \infty}(x)=U_{u}(x)$ for every $x>0$, and (ii) follows. Finally, (22) and (i) imply that $\left(0, x_{\ell, 1}\left[v_{\ell, \infty}\right]\right] \cup\left[x_{\ell, 1}\left[v_{\ell, \infty}\right], \infty\right)=\left\{x>0 ; v_{\ell, \infty}(x) \leq h(x)\right\} \subseteq\{x>0$; $\left.v_{u, \infty}(x) \leq h(x)\right\}=\left(0, x_{u, 1}\left[v_{u, \infty}\right]\right] \cup\left[x_{u, 1}\left[v_{u, \infty}\right], \infty\right)$. Hence, $0<x_{\ell, 1}\left[v_{\ell, \infty}\right] \leq x_{u, 1}\left[v_{u, \infty}\right]<$ $L<x_{u, 2}\left[v_{u, \infty}\right] \leq x_{\ell, 2}\left[v_{\ell, \infty}\right]<\infty$. Similarly, $\left(0, x_{\ell, 1}\left[v_{\ell, n}\right]\right] \cup\left[x_{\ell, 1}\left[v_{\ell, n}\right], \infty\right)=\{x>0 ;$ $\left.v_{\ell, n+1}(x) \leq h(x)\right\} \subseteq\left\{x>0 ; v_{u, n+1}(x) \leq h(x)\right\}=\left(0, x_{u, 1}\left[v_{u, n}\right]\right] \cup\left[x_{u, 1}\left[v_{u, n}\right], \infty\right)$, which implies that $0<x_{\ell, 1}\left[v_{\ell, n}\right] \leq x_{u, 1}\left[v_{u, n}\right]<L<x_{u, 2}\left[v_{u, n}\right] \leq x_{\ell, 2}\left[v_{\ell, n}\right]<\infty$ for every finite $n \geq 0$. 

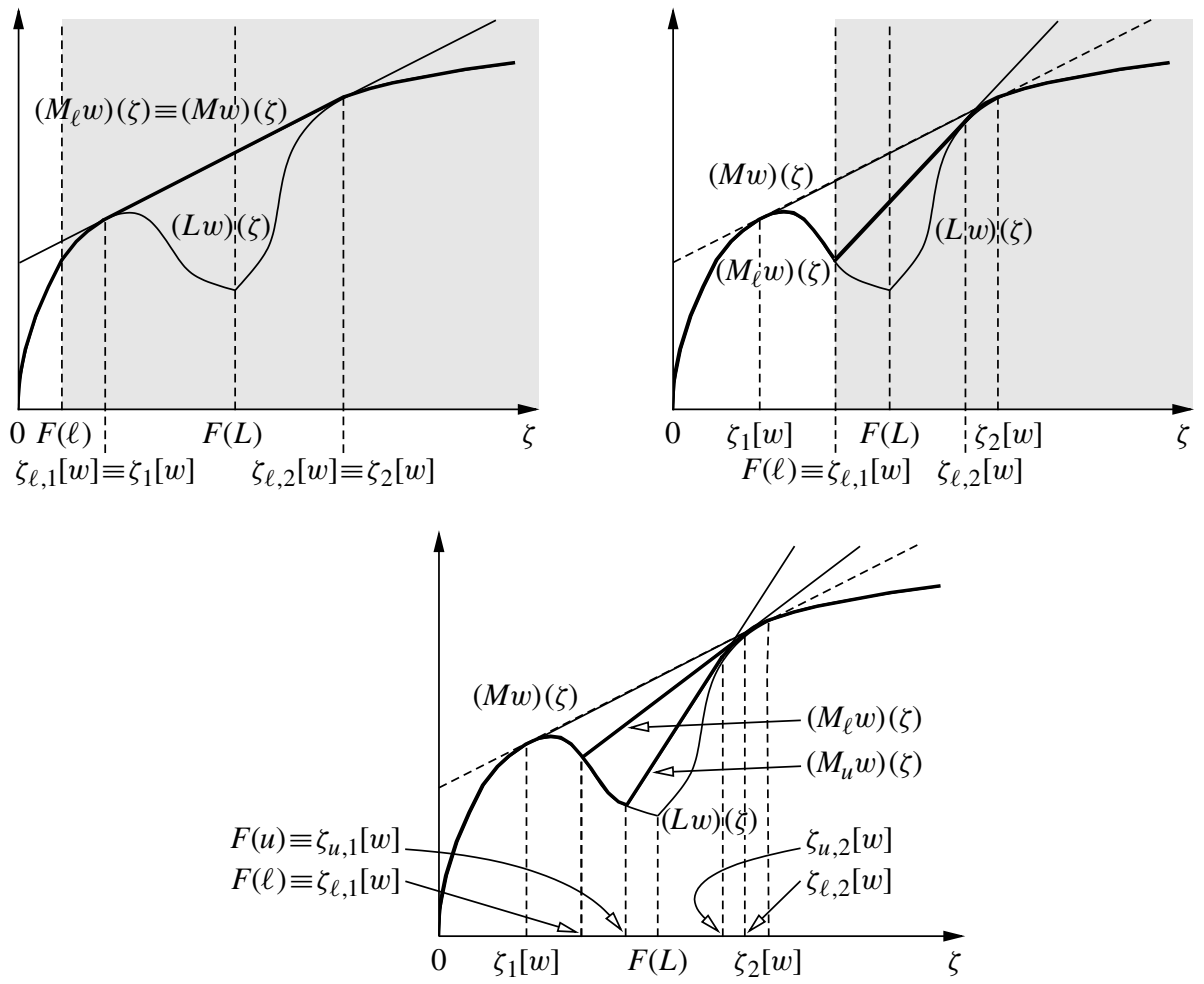

Figure 2: Sketches of $(L w)(\cdot)$ and $\left(M_{\ell} w\right)(\cdot)$. Top left: $F(\ell) \leq \zeta_{1}[w]$. Top right: $\zeta_{1}[w]<F(\ell) \leq F(L)$. Bottom: the comparison of $\left(M_{\ell} w\right)(\cdot)$ and $\left(M_{\ell} w\right)(\cdot)$ for $0<\ell<u<L$.

\section{Numerical illustration}

For illustration, we take $L=1, \sigma=0.275, r=0.03, c=0.05, \delta=0.08, \lambda \gamma=0.01$, and $y_{0}=0.03$. Observe that $0<r<c<\delta$. We obtain $\alpha_{0}=-0.3910$ and $\alpha_{1}=$ 2.7054. We implemented the successive approximations of Sections 3 and 4 in $\mathrm{R}$ in order to use readily available routines to calculate the smallest nonnegative concave majorants of functions. Specifically, we used the $\mathrm{gcml} \mathrm{cm}$ function from the R package $f d r t o o l$ developed by Korbinian Strimmer. The approximation functions approxfun and splinefun were also useful to compactly represent the functions we evaluated on appropriate grids placed on the state space and their $F$-transformations. By trial and error, we found that the optimal continuation region lies strictly inside $[0,10 L]$. Because $F(L)$ turns out to be significantly smaller than the upper bound $10 L$, for the accuracy of the results, it proved useful to put a grid over $[0, F(L)]$ that was one hundred times finer than the grid over $[F(L), F(10 L)]$.

In the implementation of the successive approximations of Sections 3 and 4, we decided to stop the iterations as soon as the maximum absolute difference between the last two approximations over the grid placed on $[0,10 L]$ is less than 0.01 . We obtained a good approximation for the first problem after three iterations with the maximum absolute difference $\left\|v_{3}-v_{2}\right\| \approx 0.0011$ and returns $v_{3}(\cdot),\left(0, x_{1}\left[v_{2}\right]\right] \cup\left[x_{2}\left[v_{2}\right], \infty\right)=(0,0.3874] \cup[4.7968, \infty)$, and $\tilde{\tau}\left[v_{3}\right]=\inf \{t \geq$ $\left.0: X_{t} \notin(0,0.3874] \cup[4.7968, \infty)\right\}$ as the approximate value function, approximate stopping region, and nearly optimal stopping rule for (5), respectively. The bound of Corollary 1 also 
guarantees that

$$
\left\|V(\cdot)-v_{3}(\cdot)\right\| \leq \frac{c L}{r}\left(\frac{\lambda \gamma}{r+\lambda \gamma}\right)^{3}=0.026 .
$$

The top-left diagram of Figure 3 suggests that the algorithm actually converges faster than what this upper bound implies. The top-middle and top-right diagrams illustrate how the solution of
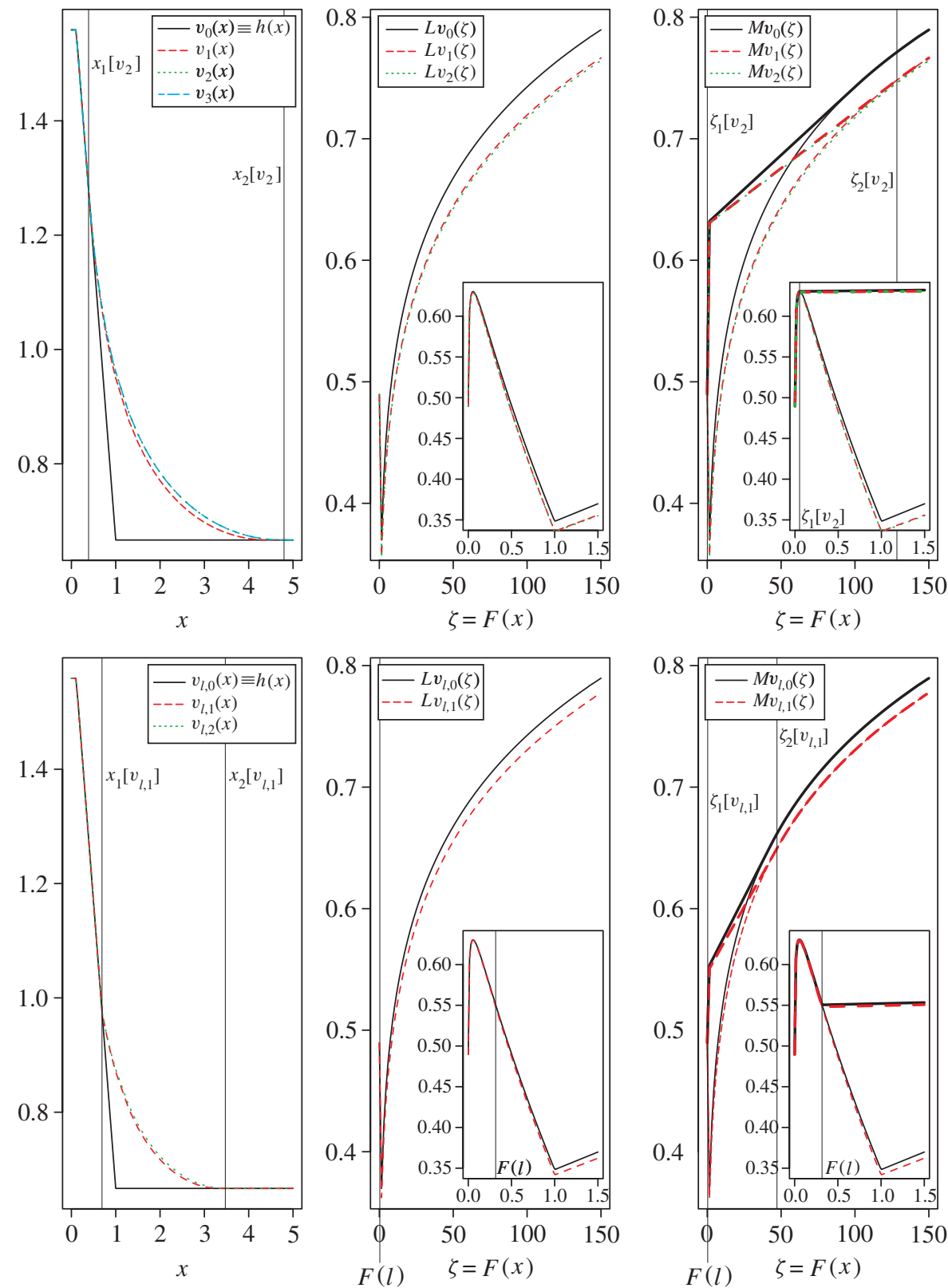

FIGURE 3: Numerical illustrations of the solutions of the auxiliary optimal stopping problems (5) (top) and (19) (bottom) for the asset manager's first and second problems (with $\ell=0.69$ ), respectively. 
each auxiliary problem is found by constructing the smallest nonnegative concave majorants $M$ of the transformations with operator $L$. The insets display zoomed-in views over the small interval $[0, F(L)]$. The top three diagrams of Figure 3 are consistent with the general form sketched in Figure 1.

The bottom three diagrams of Figure 3 similarly illustrate the solution of the second problem of the asset manager when the investors hold a limited protection of their assets with lower bound $\ell=0.69$ on the market value of the asset manager's portfolio. Because $x_{1}\left[v_{\infty}\right] \approx$ $x_{1}\left[v_{2}\right]=0.3874<\ell<4.7968=x_{2}\left[v_{2}\right] \approx x_{2}\left[v_{\infty}\right]$, the unconstrained solution of problem 1 (corresponding to $\ell=0$ ) is not optimal any more. Therefore, we calculate the successive approximations of Section 4 , which converge in two iterations because $\left\|v_{\ell, 2}-v_{\ell, 1}\right\| \approx$ $0.0063<1 / 100$. Hence, $v_{\ell, 2}(\cdot),\left(0, x_{\ell, 1}\left[v_{\ell, 1}\right]\right] \cup\left[x_{\ell, 2}\left[v_{\ell, 1}\right], \infty\right)=(0,0.69] \cup[3.4724, \infty)$, and $\tilde{\tau}_{\ell}\left[v_{\ell, 1}\right]=\inf \left\{t \geq 0: X_{t} \notin(0,0.69] \cup[3.4724, \infty)\right\}$ are the approximate value function, the stopping region, and the nearly optimal stopping rule for (19).

Observe that the stopping region of problem 2 contains the stopping region of problem 1 : $\left(0, x_{\ell, 1}\left[v_{\ell, 1}\right]\right] \cup\left[x_{\ell, 2}\left[v_{\ell, 1}\right], \infty\right)=(0,0.69] \cup[3.4724, \infty) \supset\left(0, x_{1}\left[v_{2}\right]\right] \cup\left[x_{2}\left[v_{2}\right], \infty\right)=$ $(0,0.3874] \cup[4.7968, \infty)$. Thus, the asset manager stops early in the presence of portfolio protection at level $\ell=0.69$. Because $U(x) \approx x-c L / r+v_{2}(x)$ and $U_{\ell}(x) \approx x-c L / r+v_{\ell, 1}(x)$ are approximately the value functions of problems 1 and 2 , the value of the limited protection at level $\ell$ when the stock price is $x$ equals $U(x)-U(\ell)(x) \approx v_{3}(x)-v_{\ell, 2}(x)$, which is plotted in the top diagram of Figure 4. Therefore, the no-difference price of this protection at the initiation of the contract equals $U(L)-U(\ell)(L) \approx v_{3}(L)-v_{\ell, 2}(L)=0.087$. The bottom diagram of Figure 4 shows the no-difference prices of the protection at levels $\ell$ changing between 0 and $L=1$. The protection has no value at protection levels less than or equal to $x_{1}\left[v_{\infty}\right] \approx x_{1}\left[v_{2}\right]$,
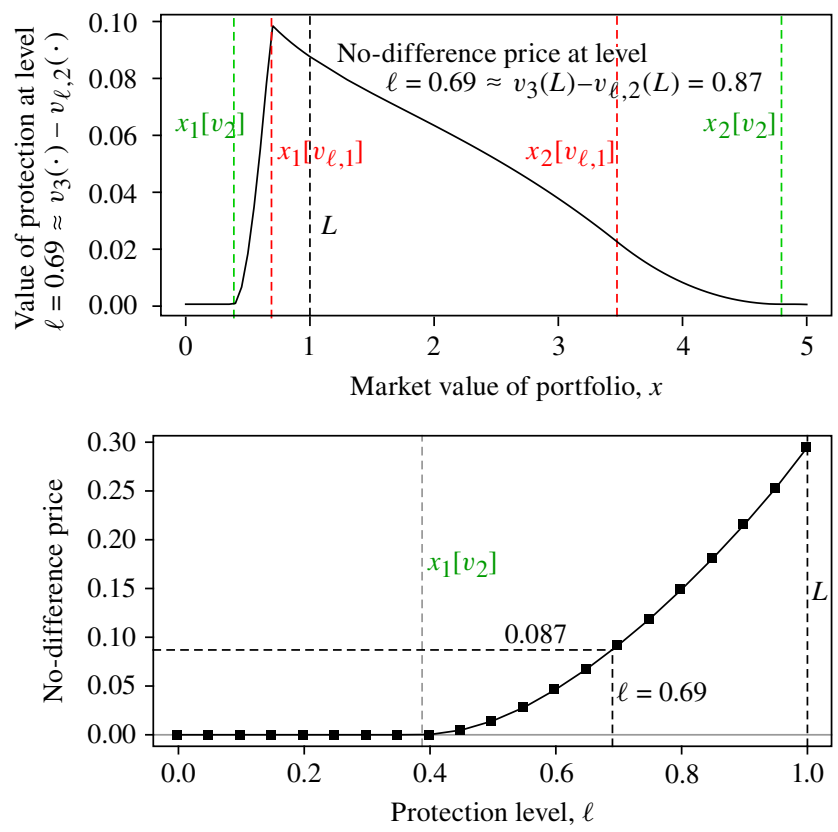

FIGURE 4: Top: the value of the limited protection at level $\ell=0.69$ as the market value of the portfolio changes. Bottom: no-difference prices of the protections for different protection limits. 
because the optimal policy, even in the absence of a protection clause, instructs the asset manager to quit as soon as the market value of the portfolio goes below $x_{1}\left[v_{\infty}\right] \approx x_{1}\left[v_{2}\right]$.

Let us finish with a final remark about the role of $L$. Let us replace $U(\cdot)$ in (2) with $U_{L}(\cdot)$ to emphasize its dependence on $L>0$. Then

$$
\begin{aligned}
U_{L}(x) & =\sup _{\tau \in S} \mathrm{E}_{x}^{\gamma}\left[\mathrm{e}^{-r \tau}\left(X_{\tau}-L\right)^{+}+\int_{0}^{\tau} \mathrm{e}^{-r t}\left(\delta X_{t}-c L\right) \mathrm{d} t\right] \\
& =\sup _{\tau \in S} L \mathrm{E}_{x}^{\gamma}\left[\mathrm{e}^{-r \tau}\left(\frac{X_{\tau}}{L}-1\right)^{+}+\int_{0}^{\tau} \mathrm{e}^{-r t}\left(\frac{\delta X_{t}}{L}-c\right) \mathrm{d} t\right] \\
& =\sup _{\tau \in S} L \mathrm{E}_{x / L}^{\gamma}\left[\mathrm{e}^{-r \tau}\left(X_{\tau}-1\right)^{+}+\int_{0}^{\tau} \mathrm{e}^{-r t}\left(\delta X_{t}-c\right) \mathrm{d} t\right] \\
& =L U_{1}\left(\frac{x}{L}\right)
\end{aligned}
$$

for every $x>0$. Therefore, we can in fact choose $L=1$ in (2) without loss of generality and solve it for $U_{1}(\cdot)$, obtaining the solutions for all other $L>0$ values by the transformation $U_{L}(x)=L U_{1}(x / L)$ for every $x>0$.

\section{Acknowledgements}

Savas Dayanik's research was partly supported by the TÜBITAK Research Grants 109M714 and 110M610. Masahiko Egami was supported in part by Grant-in-Aid for Scientific Research (B), no. 22330098, Japan Society for the Promotion of Science. The authors thank two anonymous referees and the editors for suggestions that improved the presentation of the paper.

\section{References}

[1] Asmussen, S., Avram, F. and Pistorius, M. R. (2004). Russian and American put options under exponential phase-type Lévy models. Stoch. Process. Appl. 109, 79-111.

[2] Borodin, A. N. And Salminen, P. (2002). Handbook of Brownian Motion-Facts and Formulae, 2nd edn. Birkhäuser, Basel.

[3] Boyarchenko, S. I. And LevendorskiǏ, S. Z. (2002). Perpetual American options under Lévy processes. SIAM J. Control Optimization 40, 1663-1696.

[4] Çrnlar, E. (2006). Jump-diffusions. Blackwell-Tapia Conference, 3-4 November 2006. Avalaible at http:// Www.ima.umn.edu/2006-2007/SW11.3-4.06/abstracts.html\#Cinlar-Erhan.

[5] Chan, T. (1999). Pricing contingent claims on stocks driven by Lévy processes. Ann. Appl. Prob. 9, 504-528.

[6] Colwell, D. B. And Elliott, R. J. (2006). Discontinuous asset prices and non-attainable contingent claims. Math. Finance 3, 295-308.

[7] Cont, R. And Tankov, P. (2004). Financial Modelling with Jump Processes. Chapman \& Hall/CRC, Boca Raton, FL.

[8] Davis, M. H. A. (1984). Piecewise-deterministic Markov processes: a general class of nondiffusion stochastic models. J. R. Statist. Soc. Ser. B 46, 353-388.

[9] Davis, M. H. A. (1993). Markov Models and Optimization (Monogr. Statist. Appl. Prob. 49). Chapman \& Hall, London.

[10] Dayanik, S. (2008). Optimal stopping of linear diffusions with random discounting. Math. Operat. Res. 33, 645-661.

[11] Dayanik, S. and Karatzas, I. (2003). On the optimal stopping problem for one-dimensional diffusions. Stoch. Process. Appl. 107, 173-212.

[12] Dayanik, S. and Sezer, S. (2009). Multisource Bayesian sequential hypothesis testing. Preprint.

[13] Dayanik, S., Poor, H. V. and Sezer, S. O. (2008). Multisource Bayesian sequential change detection. Ann. Appl. Prob. 18, 552-590. 
[14] Di Nunno, G., ØKsendal, B. And Proske, F. (2009). Malliavin Calculus for Lévy Processes with Applications to Finance. Springer, Berlin.

[15] Duffie, D. (1996). Dynamic Asset Pricing Theory. Princeton University Press.

[16] Duffie, D. AND GÂRLEAnU, N. (2001). Risk and valuation of collateralized debt obligations. Financial Anal. J. 57, 41-59.

[17] Duffie, D. And Singleton, K. J. (1999). Modeling term structures of defaultable bonds. Rev. Financial Stud. 12, 687-720.

[18] Egami, M. and Esteghamat, K. (2006). An approximation method for analysis and valuation of credit correlation derivatives. J. Banking Finance 30, 341-364.

[19] Hull, J. AND White, A. (2004). Valuation of a CDO and an $n^{\text {th }}$ to default CDS without Monte Carlo simulation. J. Derivatives 12, 8-23.

[20] Karlin, S. and Taylor, H. M. (1981). A Second Course in Stochastic Processes. Academic Press, New York.

[21] Kou, S. G. And Wang, H. (2004). Option pricing under a double exponential jump diffusion model. Manag. Sci. 50, 1178-1192.

[22] Leland, H. E. (1994). Corporate debt value, bond covenants, and optimal capital structure. J. Finance 49, 1213-1252.

[23] Lucas, D. J., Goodman, L. S. and Fabozzi, F. J. (2006). Collateralized Debt Obligations: Structures and Analysis. John Wiley, Hoboken, NJ.

[24] MoRDECKI, E. (1999). Optimal stopping for a diffusion with jumps. Finance Stoch. 3, 227-236.

[25] MordecKI, E. (2002). Optimal stopping and perpetual options for Lévy processes. Finance Stoch. 6, $473-493$.

[26] Pham, H. (1997). Optimal stopping, free boundary, and American option in a jump-diffusion model. Appl. Math. Optimization 35, 145-164.

[27] Schoutens, W. (2003). Lévy Processes in Finance: Pricing Financial Derivatives. John Wiley, Chichester. 